ESAIM: COCV 20 (2014) 957-982

DOI: $10.1051 / \mathrm{cocv} / 2014003$
ESAIM: Control, Optimisation and Calculus of Variations

www.esaim-cocv.org

\title{
ON ASYMPTOTIC EXIT-TIME CONTROL PROBLEMS LACKING COERCIVITY*
}

\author{
M. MotTA $^{1}$ And C. SARTORI ${ }^{1}$
}

\begin{abstract}
The research on a class of asymptotic exit-time problems with a vanishing Lagrangian, begun in [M. Motta and C. Sartori, Nonlinear Differ. Equ. Appl. Springer (2014).] for the compact control case, is extended here to the case of unbounded controls and data, including both coercive and non-coercive problems. We give sufficient conditions to have a well-posed notion of generalized control problem and obtain regularity, characterization and approximation results for the value function of the problem.
\end{abstract}

Mathematics Subject Classification. 49J15, 49N25, 93C10, 49L20, 49L25, 93D20.

Received July 2, 2013.

Published online August 5, 2014.

\section{INTRODUCTION}

We consider the exit-time control problem

$$
\left\{\begin{array}{l}
\operatorname{minimize} \int_{0}^{t_{x}(a)} l(y(t), \alpha(t)) \mathrm{d} t \\
\dot{y}(t)=f(y, \alpha), \quad y(0)=x \\
y(t) \in \mathcal{T}^{c} \forall t<t_{x}(\alpha), \quad \quad \liminf _{t \rightarrow t_{x}^{-}(\alpha)} \mathbf{d}(y(t), \mathcal{T})=0
\end{array}\right.
$$

where $\mathcal{T} \subset \mathbb{R}^{n}$ is a non empty, closed subset, which will be called the target, $\mathcal{T}^{c}$ is its complement and $\mathbf{d}$ is the distance from it. Here $\alpha(t) \in A$, where $A \subset \mathbb{R}^{m}$ is a convex, closed cone containing the origin.

The crucial assumptions of the paper are the sign condition

$$
l(x, a) \geq 0 \quad \forall(x, a) \in \mathcal{T}^{c} \times A
$$

and the unboundedness of the control set as well as of the data functions, $f$ and $l$. Precise hypotheses and definitions will be given in Section 2.

Our main purpose is to provide sufficient conditions yielding well posedness, characterization, approximation and regularity results for the asymptotic value function, $\mathcal{V}$, of (1.1).

The term asymptotic is justified by the fact that if $l(x, a)=0$ for some $(x, a) \in \mathcal{T} \times A$, there could be minimizing trajectories asymptotically approaching the target giving rise to a finite cost, even with an infinite

Keywords and phrases. Exit-time problems, impulsive optimal control problems, viscosity solutions, asymptotic controllability.

* This research is partially supported by the Marie Curie ITN SADCO, FPr-PEOPLE-2010-ITN n. 264735-SADCO and by the MIUR grant PRIN 2009 "Metodi di viscosità, geometrici e di controllo per modelli diffusivi nonlineari" (2009KNZ5FK).

1 Dipartimento di Matematica, Via Trieste, 63 - 35121 Padova, Italy. motta@math.unipd.it; sartori@math.unipd.it 
exit-time. Not surprisingly, the boundary value problem, associated to the Hamilton-Jacobi equation

$$
\mathcal{H}(x, p) \doteq \sup _{a \in A}\{-\langle p, f(x, a)\rangle-l(x, a)\}=0
$$

(see for more details (BVP) in (4.1)), has not a unique solution even among the continuous and nonnegative functions and in the compact control case studied in [16]. Indeed we can expect that $\mathcal{V}$ is the maximal subsolution to (BVP), but not the minimal nonnegative supersolution, which in general turns out to be a different value function, $V^{m}$, where also controls that do not steer to the target are admissible. The question is therefore which sufficient conditions is reasonable to introduce in order to get uniqueness, at least in several problems arising from applications. Moreover, the continuity of $\mathcal{V}$ on the target is not enough to yield the continuity on its domain and the value functions of regular approximations of the cost may not converge to $\mathcal{V}$.

Besides the questions related to the degeneracy of the Lagrangian, the main issue is now the unboundedness of the data, which gives rise to a whole new set of problems. In dealing with $A$ unbounded it is often assumed that $f$ has polynomial growth of power $p \geq 1$ in the control variable $a$ and that

$$
l(x, a) \geq C_{2}|a|^{q}-C_{1} \quad \forall(x, a) \in \mathbb{R}^{n} \times A,
$$

for some constants $C_{1}>0, C_{2} \geq 0$ and $q \geq p$. Condition (1.3) is known as coercivity if $q>p$ and as weak coercivity when $q=p$. For the finite horizon case, (1.3) for $q>p$ yields a suitable compactness property for admissible controls, while for $q=p$ the natural framework is that of generalized controls. Since we have an exittime problem, we introduce the following weaker version of (1.3), called in the sequel target-weighted coercivity $($ weak, if $q=p)$ :

$$
l(x, a) \geq c_{2}(\mathbf{d}(x))|a|^{q}-C_{1} \quad \forall(x, a) \in \mathcal{T}^{c} \times A,
$$

where $C_{1} \geq 0$ and $\left.c_{2}:\right] 0,+\infty[\rightarrow] 0,+\infty[$ is a continuous, increasing function. We prove several results assuming either (1.3) or (1.4), but we cover cases, such as the so-called cheap control problems, where for instance $l$ may depend only on $x$, in which neither (1.3) nor (1.4) holds.

As a consequence, it could be optimal to implement controls with energy blow-ups, in the sense that the energy, $\int_{0}^{t_{x}(\alpha)}|\alpha(t)|^{q} \mathrm{~d} t$, is equal to $+\infty$ for $t_{x}(\alpha)<+\infty$ (see for instance Example 3.10). Moreover, minimizing sequences of trajectories may converge to a discontinuous function. Hence we embed our problem in an extended setting, where measures act as controls. To this end, we follow the graph-completion approach introduced in [4], as developed in [17] (see also $[3,11]$ and the references therein). By the very nature of the problem, minimizing controls in our case lack the two key properties, that is finite energy or horizon, which either together or singularly, are generally used in the literature to introduce a proper extension for several problems. In those cases, the infimum of the cost over extended controls was the same as over original controls. A careful choice of the set of admissible controls leads us to introduce a wider class of extended controls, which fits the generality of our problem and is proper, at least if the value function $\mathcal{V}$ is continuous on the target.

Furthermore, in Section 4 we thoroughly investigate the important issue of the characterization of the value function. Using optimality principles, we prove that $\mathcal{V}$, when continuous on the target, is the maximal subsolution of (BVP), and derive a representation formula for the minimal, nonnegative supersolution of (BVP), $V^{m}$. As a consequence, we characterize $\mathcal{V}$ as the unique non negative solution of (BVP) by imposing that $V^{m} \equiv \mathcal{V}$. Then we introduce sufficient conditions ensuring such an equality, satisfied in many applications and generalizing previous hypotheses (for an overview on known results and some applications we refer to Sect. 5 of [12], to [10], and to the references therein). In particular, our hypotheses lead to obtain uniqueness even for some weakly coercive and some cheap control problems.

Finally we extend the regularity and approximation results for $\mathcal{V}$ obtained in [16] in the case of $A$ compact to $A$ unbounded and in both coercive and non coercive problems. We provide several examples to better illustrate the main topics.

Some last comments. Many infinite horizon problems may be seen as asymptotic exit-time problems by choosing a suitable target. For instance, the infinite horizon LQR problem and some further generalizations, as well as singular perturbation of cheap control problems, are included setting, generally, $\mathcal{T} \equiv\{0\}$. 
For the uniqueness issue, in the case of a nonnegative Lagrangian there is a wide bibliography, mostly concerning the case of bounded controls, as recalled in [16]. Some optimality principles were stated for the coercive case in $[9,18]$ and introduced for weakly coercive problems in [12]. We recall also the paper [6], where a coercive infinite dimensional problem is considered.

The paper is organized as follows. In Section 2 we state the precise formulation of the problem, and recall some results from [14], which provide the construction of trajectories approaching asymptotically the target with finite cost. In Section 3 we introduce the generalized control problem and investigate the well-posedness issue. Refining the optimality principles in [12], in Section 4 we obtain a uniqueness theorem for the solution of (BVP). Section 5 is devoted to the approximation and regularization problem. In Section 6 we introduce explicit conditions, sufficient to propagate the continuity of $\mathcal{V}$ from $\partial \mathcal{T}$. The Appendix, containing the proof of a density result, concludes the paper.

Notations. Let $D \subset \mathbb{R}^{N}$ for some $N \in \mathbb{N}$. $\forall r>0$ we denote by $D_{r}$ the closed set $\overline{B(D, r)}$, while $D_{r}^{c}=\mathbb{R}^{N} \backslash D_{r}$. $\stackrel{\circ}{D}$ is the interior of $D$. Moreover, $\chi_{D}$ denotes the characteristic function of $D$, namely for any $x \in \mathbb{R}^{N}$ we set $\chi_{D}(x)=1$ if $x \in D$ and $\chi_{D}(x)=0$ if $x \notin D$. For any function $u: \mathbb{R}^{n} \backslash \stackrel{\circ}{\mathcal{T}} \rightarrow R \cup\{+\infty\}$, we denote the set $\left\{x \in \mathbb{R}^{n} \backslash \stackrel{\circ}{\mathcal{T}}: u(x)<+\infty\right\}$ by $\operatorname{Dom}(u)$. $\left[0,+\infty\left[\doteq \mathbb{R}_{+}\right.\right.$. A function $\omega: \mathbb{R}_{+} \times \mathbb{R}_{+} \rightarrow \mathbb{R}_{+}$is called a modulus if: $\omega(\cdot, R)$ is increasing in a neighborhood of 0 , continuous at 0 , and $\omega(0, R)=0$ for every $R>0 ; \omega(r, \cdot)$ is increasing for every $r$. Let $\Omega \supset \mathcal{T}$ be an open set and let $U: \Omega \backslash \stackrel{\circ}{\mathcal{T}} \rightarrow \mathbb{R}_{+}$be a locally Lipschitz function. Then $D^{*} U(x) \doteq\left\{p \in \mathbb{R}^{n}: p=\lim _{k} \nabla U\left(x_{k}\right), x_{k} \in \operatorname{diff}(U) \backslash\{x\}, \lim _{k} x_{k}=x\right\}$ is the set of limiting gradients of $U$ at $x$ (here $\nabla$ denotes the gradient operator and $\operatorname{diff}(U)$ is the set of differentiability points of $U$ ). $U$ is said positive definite on $\Omega \backslash \mathcal{T}$ if $U(x)>0 \forall x \in \Omega \backslash \mathcal{T}$ and $U(x)=0 \forall x \in \partial \mathcal{T}$. $U$ is called proper on $\Omega \backslash \mathcal{T}$ if $U^{-1}(K)$ is compact for every compact set $K \subset \mathbb{R}_{+}$. For the notion of locally semiconcave function and of viscosity solution we refer e.g. to $[2,7]$. $\mathcal{K} \mathcal{L}$ denotes the set of all continuous functions $\beta: \mathbb{R}_{+} \times \mathbb{R}_{+} \rightarrow \mathbb{R}_{+}$such that: (1) $\beta(0, t)=0$ and $\beta(\cdot, t)$ is strictly increasing and unbounded for each $t \geq 0 ;(2) \beta(r, \cdot)$ is decreasing for each $r \geq 0$; (3) $\beta(r, t) \rightarrow 0$ as $t \rightarrow+\infty$ for each $r \geq 0$.

\section{Exit-Time PROBlems With BLOW-UP}

\subsection{Basic assumptions}

The following general hypotheses (H0) and (H1) will be assumed throughout the whole paper.

(H0) The target set $\mathcal{T} \subset \mathbb{R}^{n}$ is nonempty, closed, and with compact boundary.

The control set $A \subset R^{m}$ is a convex, closed, nontrivial cone containing the origin.

The functions $f: \mathbb{R}^{n} \times A \rightarrow \mathbb{R}^{n}, l: \mathbb{R}^{n} \times A \rightarrow \mathbb{R}$ are continuous, there exist $p, q \in \mathbb{N}, q \geq p \geq 1$, $M>0$, and for any $R>0$ there are $L_{R}, M_{R}>0$ and a modulus $\omega(\cdot, R)$, such that $\forall x, x_{1}, x_{2} \in \mathbb{R}^{n}$, $\forall a \in A$,

$$
\begin{aligned}
\left|f\left(x_{1}, a\right)-f\left(x_{2}, a\right)\right| & \leq L_{R}\left(1+|a|^{p}\right)\left|x_{1}-x_{2}\right|, \\
\left|l\left(x_{1}, a\right)-l\left(x_{2}, a\right)\right| & \leq\left(1+|a|^{q}\right) \omega\left(\left|x_{1}-x_{2}\right|, R\right) \\
0 & \leq l(x, a) \leq M_{R}\left(1+|a|^{q}\right) \quad \text { if }\left|x_{1}\right|,\left|x_{2}\right|,|x| \leq R, \\
|f(x, a)| & \leq M\left(1+|a|^{p}\right)(1+|x|) .
\end{aligned}
$$

(H1) Let $\Phi \in\{f, l\}$. There exists a continuous function $\Phi^{\infty}$, called the recession function of $\Phi$, verifying

$$
\lim _{\rho \rightarrow 0^{+}} \rho^{q} \Phi\left(x, \rho^{-1} a\right) \doteq \Phi^{\infty}(x, a)
$$

uniformly on compact sets of $R^{n} \times A$.

(H1) is a regularity hypothesis in the control variable at infinity, essential in order to introduce the generalized problem associated to (1.1) (see Sect. 3). 
Example 2.1. Functions $f$ and $l$ which are polynomials in the control variable $a$, satisfy (H1). If, for instance, there are some continuous functions $f_{i}, F_{i, j}$ such that

$$
f(x, a)=f_{0}(x)+\sum_{i=1}^{m} f_{i}(x) a_{i}+\sum_{i, j=1}^{m} F_{i, j}(x) a_{i} a_{j} \quad \forall(x, a) \in \mathbb{R}^{n} \times A,
$$

then $p=2$ and $f^{\infty}(x, a)=\sum_{i, j=1}^{m} F_{i, j}(x) a_{i} a_{j}$ if $q=2$, while $f^{\infty}(x, a) \equiv 0$ if $q>2$.

Notice that if $q>p$, then one always has $f^{\infty} \equiv 0$.

\subsection{Optimization problems}

We consider a nonlinear control system having the form

$$
\dot{y}(t)=f(y(t), \alpha(t)), \quad y(0)=x \quad\left(x \in \mathbb{R}^{n}\right)
$$

and a payoff

$$
\mathcal{J}(t, x, \alpha)=\int_{0}^{t} l(y(s), \alpha(s)) \mathrm{d} s .
$$

The controls $\alpha$ are assumed to belong to the set

$$
\mathcal{A} \doteq\left\{\alpha \in \mathcal{B} \cap L_{\mathrm{loc}}^{q}\left(\left[0, T_{\alpha}[, A) \text { for some } T_{\alpha} \in\right] 0,+\infty\right] \quad \text { and } \quad \int_{0}^{T_{\alpha}}|\alpha(t)|^{q} \mathrm{~d} t=+\infty \quad \text { if } T_{\alpha}<+\infty\right\},
$$

where $\mathcal{B}$ denotes the set of the Borel-measurable functions. $T_{\alpha}$, if finite, will be called the (energy) blow-up time. If the growth of $l$ in $a$ is of order less than $p$, or $l$ does not depend on $a$, we assume $q=p$. We refer to Remark 2.2 below for some comments on the set $\mathcal{A}$.

By hypothesis (H0), for any $x \in \mathbb{R}^{n}$ and for any control $\alpha \in \mathcal{A}$, the control system in (2.3) admits just one solution defined on the interval $\left[0, T_{\alpha}\right.$ [ and the corresponding payoff is surely defined for all $t \in\left[0, T_{\alpha}[\right.$. We use $y_{x}(\cdot, \alpha)$ (or, when no confusion arises, $\left.y_{x}(\cdot)\right)$ to denote such a solution.

Let us write two estimates, useful in the sequel, that can be obtained by standard tools. For every $x, z \in \mathbb{R}^{n}$, $\forall \alpha \in \mathcal{A}$, and $\forall t \in\left[0, T_{\alpha}[\right.$ one has

$$
\left|y_{x}(t, \alpha)\right| \leq\left(|x|+M t+M \int_{0}^{t}\left|\alpha\left(t^{\prime}\right)\right|^{p} \mathrm{~d} t^{\prime}\right) \mathrm{e}^{M\left(t+\int_{0}^{t}\left|\alpha\left(t^{\prime}\right)\right|^{p} \mathrm{~d} t^{\prime}\right)}
$$

and, if $\exists R>0$ such that $\left|y_{x}\left(t^{\prime}, \alpha\right)\right|,\left|y_{z}\left(t^{\prime}, \alpha\right)\right| \leq R \forall t^{\prime} \in[0, t]$, then

$$
\left|y_{x}(t, \alpha)-y_{z}(t, \alpha)\right| \leq|x-z| \mathrm{e}^{L_{R}\left(t+\int_{0}^{t}\left|\alpha\left(t^{\prime}\right)\right|^{p} \mathrm{~d} t^{\prime}\right)} .
$$

For any $x \in \mathcal{T}^{c}$ and $\alpha \in \mathcal{A}$, we set

$$
t_{x}(\alpha) \doteq \inf \left\{t \in \left[0, T_{\alpha}\left[: \quad y_{x}(t, \alpha) \in \mathcal{T}\right\} \quad\left(\leq T_{\alpha}\right) .\right.\right.
$$

Notice that $t_{x}(\alpha)$ is an effective exit-time from $\mathcal{T}^{c}$ only in case $t_{x}(\alpha)<T_{\alpha}$. Otherwise, $t_{x}(\alpha)=T_{\alpha}$. Since the zero-level set of $l$, defined as follows,

$$
\mathcal{Z} \doteq\{x: l(x, a)=0 \text { for some } a \in A\}
$$

may be not empty and we do not assume coercivity, we may have finite cost even if $t_{x}(\alpha)=T_{\alpha}$ and $T_{\alpha} \leq+\infty$. It is therefore very natural to consider the subset of admissible controls

$$
\mathcal{A}(x) \doteq\left\{\alpha \in \mathcal{A}: \liminf _{t \rightarrow t_{x}^{-}(\alpha)} \mathbf{d}\left(y_{x}(t, \alpha)\right)=0\right\}
$$


and the (asymptotic) exit-time value function

$$
\mathcal{V}(x) \doteq \inf _{\alpha \in \mathcal{A}(x)} \mathcal{J}\left(t_{x}(\alpha), x, \alpha\right) \quad(\leq+\infty) .
$$

For the characterization of $\mathcal{V}$ that we will discuss in Section 4, it is useful to introduce the following minimal value function

$$
\mathcal{V}^{m}(x) \doteq \inf _{\alpha \in \mathcal{A}} \mathcal{J}\left(t_{x}(\alpha), x, \alpha\right),
$$

where also non asymptotic controls with either finite or infinite blow up time are allowed.

Remark 2.2. The choice of the admissible control set $\mathcal{A}$ is a delicate point. If we assumed either coercivity or weak coercivity, i.e. (1.3) for $q \geq p$, it would be not a restriction to allow only controls in $L_{\text {loc }}^{q}\left(\mathbb{R}_{+}, A\right)$. Condition (1.3) implies indeed that

$$
\mathcal{J}(t, x, \alpha) \geq C_{2} \int_{0}^{t}|\alpha(\tau)|^{q} \mathrm{~d} \tau-C_{1} t \quad \forall t>0,
$$

hence for controls $\alpha \in \mathcal{A}$ such that

$$
t_{x}(\alpha)=T_{\alpha}<+\infty \quad \text { and } \quad \int_{0}^{T_{\alpha}}|\alpha(t)|^{q} \mathrm{~d} t=+\infty,
$$

we will never obtain an optimal cost. Nevertheless, by the generality of our problem, we cannot avoid to consider minimizing sequences of controls with finite blow-up times (see for instance Example 3.10).

As we will show in Section 5, the minimization of the cost $\mathcal{J}$ over some control subsets of $\mathcal{A}(x)$, say $\tilde{\mathcal{A}}(x)$, gives rise again to the same $\mathcal{V}$ as soon as the function

$$
\mathcal{U}^{\tilde{\mathcal{A}}}(x) \doteq \inf _{\alpha \in \tilde{\mathcal{A}}(x)} \mathcal{J}\left(t_{x}(\alpha), x, \alpha\right)
$$

is continuous on $\partial \mathcal{T}$. For instance, $\tilde{\mathcal{A}}(x)$ can include just controls of $\mathcal{A}(x)$ in $L_{\text {loc }}^{r}\left(\mathbb{R}_{+}, A\right)$, or in $L^{r}\left(\mathbb{R}_{+}, A\right)$ for some integer $r \geq q$, or controls $\alpha$ such that $t_{x}(\alpha)<+\infty$, (see Thm. 5.5 and Rem. 5.6).

\subsection{Known results}

Let us now recall some notions and results by [14], useful in the sequel. For some terminology borrowed from non smooth analysis we refer to the Notations in the Introduction. In the rest of this subsection, we fix a compact control set $A^{\prime} \subset A$ and a continuous Lagrangian $h: \mathbb{R}^{n} \times A^{\prime} \rightarrow \mathbb{R}_{+}$.

Definition 2.3. [14] Given an open set $\Omega \subset \mathbb{R}^{n}, \Omega \supset \mathcal{T}$ we say that $U: \Omega \backslash \stackrel{\circ}{\mathcal{T}} \rightarrow \mathbb{R}_{+}$is a local Minimum Restraint Function, in short, a local MRF, for the pair $\left(h, A^{\prime}\right)$, if $U$ is continuous on $\Omega \backslash \stackrel{\circ}{\mathcal{T}}$, locally semiconcave, positive definite, proper on $\Omega \backslash \mathcal{T}$,

$$
\left.\left.\exists U_{0} \in\right] 0,+\infty\right]: \quad \lim _{x \rightarrow x_{0}, x \in \Omega} U(x)=U_{0} \quad \forall x_{0} \in \partial \Omega ; \quad U(x)<U_{0} \quad \forall x \in \Omega \backslash \stackrel{\circ}{\mathcal{T}},
$$

and, moreover, $\exists k>0$ such that, for every $x \in \Omega \backslash \mathcal{T}$,

$$
\min _{a \in A^{\prime}}\{\langle p, f(x, a)\rangle+k h(x, a)\}<0 \quad \forall p \in D^{*} U(x),
$$

where $D^{*} U(x)$ is the set of limiting gradients of $U$ at $x$. 
By [14] we recall that, $\forall x \in \Omega \backslash \mathcal{T}$ inequality (2.12) is equivalent to

$$
\min _{a \in A^{\prime}}\{\langle p, f(x, a)\rangle+k h(x, a)+m(U(x))\} \leq 0 \quad \forall p \in D^{*} U(x),
$$

where $m:] 0,+\infty[\rightarrow] 0,+\infty[$ is some continuous, increasing function.

The main result of [14] is the following theorem, providing a sufficient condition for local asymptotic controllability of the system and boundedness of the cost, in terms of a MRF $U$. For more details on this topic, we refer to [14].

Theorem 2.4. [14] If there exists a local $M R F U: \Omega \rightarrow \mathbb{R}_{+}$for $\left(h, A^{\prime}\right)$, then for any $\left.\sigma \in\right] 0, U_{0}$ [ there is some $\beta \in \mathcal{K} \mathcal{L}$ such that $\forall \eta>0$ and $\left.\left.\forall x \in U^{-1}(] 0, \sigma\right]\right)$, there exists a Borel-measurable control $\alpha: \mathbb{R}_{+} \rightarrow A^{\prime}$ verifying

$$
\mathbf{d}\left(y_{x}(t, \alpha)\right) \leq \beta(\mathbf{d}(x), t) \quad \forall t \in \mathbb{R}_{+}
$$

and

$$
\int_{0}^{t_{x}(\alpha)}\left[k h\left(y_{x}(t, \alpha), \alpha(t)\right)+m\left(U\left(y_{x}(t, \alpha)\right)\right)\right] \mathrm{d} t \leq(1+\eta) U(x) .
$$

Therefore the value function

$$
\mathcal{U}(x) \doteq \inf _{\alpha \in \mathcal{A}(x)} \int_{0}^{t_{x}(\alpha)} h\left(y_{x}(s, \alpha), \alpha(s)\right) \mathrm{d} s
$$

is continuous on $\partial \mathcal{T}$. In particular, if there exists a local $M R F U$ for $\left(l, A^{\prime}\right)$, then $\mathcal{V}$ is continuous on $\partial \mathcal{T}$.

Remark 2.5. The thesis of Theorem 2.4 remains unchanged if, instead of assuming the existence of a local MRF for $\left(h, A^{\prime}\right)$ for some fixed compact subset of $A$, we consider the following hypothesis:

there exists a local MRF $U$ for $(h, A)$ such that $\forall x \in \Omega \backslash \mathcal{T}$ :

$$
\frac{\min }{a \in A \cap B(0, R(U(x)))}\{\langle p, f(x, a)\rangle+k h(x, a)\}<0, \quad \forall p \in D^{*} U(x),
$$

where $R:] 0, \sigma] \rightarrow] 0,+\infty$ [ is a decreasing continuous function (in particular, we may have $\lim _{\delta \rightarrow 0^{+}} R(\delta)=+\infty$ ). With a small abuse of notation, we will refer to such a $U$ as to a local MRF for $(h, A)$.

The proof of the results in [14] can be indeed easily adapted to this case, since the dynamics $f=f(x, a)$ was supposed there merely continuous on $\mathcal{T}^{c} \times A$ (hence, possibly unbounded around the target, in spite of the compactness of the control set assumed there). More precisely, the estimates in the proof of Theorem 1.1 (and of the technical Lem. 2.1) in [14], were done for $x \in U^{-1}\left(\left[\mu_{1}, \mu_{2}\right]\right)$ for some $0<\mu_{1}<\mu_{2} \leq \sigma$, and all the resulting constants were depending on $\mu_{1}$ and $\mu_{2}$. Thus, in any "strip" $U^{-1}\left(\left[\mu_{1}, \mu_{2}\right]\right)$ we can argue in the same way, simply by replacing the fixed control set considered there by the compact set $A^{\prime} \doteq A \cap \overline{B\left(0, R\left(\mu_{1}\right)\right)}$, which satisfies (2.12) in view of (2.16).

The following proposition is stated without proof, since it easy follows by Dynamic Programming arguments, taking into account the estimates (2.5) and (2.6).

Proposition 2.6. If $\mathcal{V}$ is continuous on $\partial \mathcal{T}, \operatorname{Dom}(\mathcal{V})$ is an open set and $\mathcal{V}$ is locally bounded and upper semicontinuous in it. 


\section{Generalized problems And Well posedness}

Since we do not assume coercivity, we introduce, as usual, the impulsive or generalized setting. More precisely, following the so-called graph completion approach, we represent generalized controls and trajectories as reparametrizations (through a possibly discontinuous time-change) of controls and trajectories of the extended minimization problem below, involving bounded-valued controls (see $[4,12,17]$ and the references therein). Then we discuss the well posedness of the extended asymptotic exit-time problem, $V$, and of the extended minimal value function, $V^{m}$, candidate to be the minimal nonnegative supersolution to the boundary value problem associated to $\mathcal{V}$ (see Sect. 4). Finally, we consider the degenerate cheap control problem, where $l(x, a) \equiv l(x)$.

Let us remark that by well posedness (or equivalently by proper extension) we mean that the original and the extended problems have the same values. This result will be proved disregarding the possibility of approximating any trajectory of the extended system (3.3) below by trajectories of the original system (2.3). In fact, such a convergence is a stronger requirement, more difficult to hold in an infinite horizon problem.

Define on $\mathbb{R}^{n} \times\left(\mathbb{R}_{+} \times A\right)$ the extended dynamics and Lagrangian $\bar{f}, \bar{l}$ as follows:

$$
\bar{\Phi}\left(x, w_{0}, w\right) \doteq\left\{\begin{array}{l}
w_{0}^{q} \Phi\left(x, w_{0}^{-1} w\right) \text { if } w_{0} \neq 0 \\
\Phi^{\infty}(x, w) \text { if } w_{0}=0 .
\end{array} \quad \Phi \in\{f, l\}\right.
$$

where $\Phi^{\infty}$ is defined in (H1). $\bar{f}, \bar{l}$ are continuous, $q$-positively homogeneous in the control variable $\left(w_{0}, w\right)$ and inherit properties analogous to those of $f$ and $l$, respectively (see e.g. [12]).

Let $S(A) \doteq\left(\mathbb{R}_{+} \times A\right) \cap\left\{\left(w_{0}, w\right): w_{0}^{q}+|w|^{q}=1\right\}$. Define the set of extended controls as

$$
\Gamma \doteq\left\{\left(w_{0}, w\right): \quad\left(w_{0}, w\right) \in \mathcal{B}\left(\mathbb{R}_{+}, S(A)\right)\right\},
$$

and $\forall\left(w_{0}, w\right) \in \Gamma$ denote by $\xi(\cdot) \equiv \xi_{x}\left(\cdot, w_{0}, w\right)$ the extended trajectory solving the extended control system

$$
\xi^{\prime}(s)=\bar{f}\left(\xi(s), w_{0}(s), w(s)\right) \quad \xi(0)=x .
$$

For any $S>0$, the extended payoff is given by

$$
J\left(S, x, w_{0}, w\right)=\int_{0}^{S} \bar{l}\left(\xi(s), w_{0}(s), w(s)\right) \mathrm{d} s .
$$

In the next proposition we show that the solutions to (3.3) are simply time-reparametrizations of trajectories of (2.3) if the controls belong to the subset

$$
\Gamma^{+} \doteq \Gamma \cap\left\{\left(w_{0}, w\right): w_{0}>0 \text { a.e. }\right\}
$$

Proposition 3.1. For any $\alpha \in \mathcal{A}$ there exist a control $\left(w_{0}, w\right) \in \Gamma^{+}$and a time-reparametrization $t: \mathbb{R}_{+} \rightarrow$ $\left[0, T_{\alpha}\left[\right.\right.$ such that $y_{x}(t(\cdot), \alpha)$ is the solution of $(3.3)$ associated to the control $\left(w_{0}, w\right)$.

Vice-versa, for any control $\left(w_{0}, w\right) \in \Gamma^{+}$with $T \doteq \int_{0}^{+\infty} w_{0}^{q}(\sigma) \mathrm{d} \sigma \leq+\infty$ there exist a control $\alpha \in \mathcal{A}$ and a time-reparametrization $s:\left[0, T\left[\rightarrow \mathbb{R}_{+}\right.\right.$such that $\xi_{x}\left(s(\cdot), w_{0}, w\right)$ is the solution of (2.3) corresponding to the control $\alpha$.

Proof. For any $\alpha \in \mathcal{A}$, set $s(t) \doteq \int_{0}^{t}\left(1+|\alpha(\tau)|^{q}\right) \mathrm{d} \tau$ for all $t \in\left[0, T_{\alpha}\left[\right.\right.$ and denote with $t: \mathbb{R}_{+} \rightarrow\left[0, T_{\alpha}\right.$ [ its inverse function. Applying the chain rule one obtains that $y_{x}(t(\cdot), \alpha)$ is a solution of (3.3) associated to the control $\left(w_{0}, w\right) \in \Gamma^{+}$given by $w(\cdot) \doteq \frac{\alpha(t(\cdot))}{\left(1+\mid \alpha\left(\left.t(\cdot)\right|^{q}\right)^{1 / q}\right.}, w_{0}(\cdot) \doteq\left(1-|w(\cdot)|^{q}\right)^{1 / q}$.

Vice-versa, for any control $\left(w_{0}, w\right) \in \Gamma^{+}$, defining $t(s) \doteq \int_{0}^{s} w_{0}^{q}(\sigma) \mathrm{d} \sigma, T \doteq \int_{0}^{+\infty} w_{0}^{q}(\sigma) \mathrm{d} \sigma$ and $s:\left[0, T\left[\rightarrow \mathbb{R}_{+}\right.\right.$ as the (continuous) inverse function of $t(s)$, one has that $\xi_{x}\left(s(\cdot), w_{0}, w\right)$ is a solution of $(2.3)$ corresponding to the control $\alpha(\cdot) \doteq \frac{w(s(\cdot))}{w_{0}(s(\cdot))} \in \mathcal{A}$. 
Remark 3.2. Considering extended controls where $w_{0}(s)=0$ for $s$ in some intervals, is a way to introduce a notion of generalized control, where the (discontinuous) generalized solution to (2.3) corresponding to $\left(w_{0}, w\right)$, say $y_{x}^{g e n}$ is defined as $y_{x}^{g e n}(\cdot) \doteq \xi_{x}\left(s(\cdot), w_{0}, w\right)$, where $s(\cdot)$ is, e.g., the right inverse of $t(s) \doteq \int_{0}^{s} w_{0}^{q}(\sigma) \mathrm{d} \sigma$ for $s \geq 0$. It is clear that, for $q>p$, one has $f^{\infty} \equiv 0$ and $y_{x}^{g e n}(\cdot) \equiv y_{x}(\cdot)$ (for more details, see [17]).

Remark 3.3. In view of Remark 2.2, we notice that the set of controls $\left\{\alpha: \alpha \in \mathcal{B} \cap L_{\mathrm{loc}}^{q}\left(\mathbb{R}_{+}, A\right)\right\}$ is embedded in the subset of controls $\left(w_{0}, w\right) \in \Gamma$ such that

$$
\int_{0}^{+\infty} w_{0}^{q}(\sigma) \mathrm{d} \sigma=+\infty
$$

Let $x \in \mathcal{T}^{c}$. For any $\left(w_{0}, w\right) \in \Gamma$ the extended exit-time is given by

$$
\sigma_{x}\left(w_{0}, w\right) \doteq \inf \left\{s>0: \quad \xi_{x}\left(s, w_{0}, w\right) \in \mathcal{T}\right\} \quad(\leq+\infty) .
$$

Defined the set of extended admissible controls

$$
\Gamma(x) \doteq\left\{\left(w_{0}, w\right) \in \Gamma: \quad \liminf _{s \rightarrow \sigma_{x}^{-}\left(w_{0}, w\right)} \mathbf{d}\left(\xi_{x}\left(s, w_{0}, w\right)\right)=0\right\},
$$

the extended (asymptotic) exit-time value function is given by

$$
V(x) \doteq \inf _{\left(w_{0}, w\right) \in \Gamma(x)} J\left(\sigma_{x}\left(w_{0}, w\right), x, w_{0}, w\right) \quad(\leq+\infty) .
$$

Similarly, we define

$$
V^{m}(x) \doteq \inf _{\left(w_{0}, w\right) \in \Gamma} J\left(\sigma_{x}\left(w_{0}, w\right), x, w_{0}, w\right),
$$

where also the cost $J\left(+\infty, x, w_{0}, w\right)$ corresponding to extended trajectories remaining away from the target is allowed. Of course, $V^{m} \leq V$.

In view of Proposition 3.1, in the extended setting we can recover both $\mathcal{V}$ and $\mathcal{V}^{m}$, by restricting the minimization to $\Gamma^{+} \cap \Gamma(x)$ and $\Gamma^{+}$in the definition of $V$ and $V^{m}$, respectively. In general, however, $V \leq \mathcal{V}$ and $V^{m} \leq \mathcal{V}^{m}$ and both these and the previous inequalities may be strict, as shown in Examples 3.5 and 3.7 below.

\subsection{Well posedness of $V$}

Proposition 3.4. Let $q>p$. Then the original and the extended exit-time problem are equivalent, that is: $V \equiv \mathcal{V}$.

Proof. Let $x \in \mathcal{T}^{c}$ be such that $V(x)<+\infty$ (otherwise, $\mathcal{V}(x)=V(x)=+\infty$ ). In the case $q>p$, any control $(0, w) \in \Gamma$ does not belong to $\Gamma(x)$, since $\xi_{x}(s, 0, w)=x \notin \mathcal{T}$ for all $s \geq 0$. Thus, owing to Proposition 3.1, in order to prove that $V(x)=\mathcal{V}(x)$, it is enough to show that the payoff corresponding to any control $\left(w_{0}, w\right) \in \Gamma(x)$ such that $w_{0}=0$ on some interval $\left[s_{1}, s_{2}\right]$, is greater than or equal to the payoff associated to some control $\left(\tilde{w}_{0}, \tilde{w}\right) \in \Gamma^{+} \cap \Gamma(x)$.

In fact, in the case that $\left[s_{1}, s_{2}\right]$ is the only interval where $w_{0} \equiv 0$, the trajectory $\xi_{x}\left(s, w_{0}, w\right) \equiv \xi_{x}\left(s_{1}, w_{0}, w\right)$ for all $s \in\left[s_{1}, s_{2}\right]$ because of the definition of $f^{\infty}$, while $l \geq 0$ implies that $\int_{s_{1}}^{s_{2}} \bar{l}\left(\xi_{x}\left(s, w_{0}, w\right), w_{0}, w\right) \mathrm{d} s \geq 0$. Therefore $J\left(\sigma_{x}\left(w_{0}, w\right), x, w_{0}, w\right) \geq J\left(\sigma_{x}\left(\tilde{w}_{0}, \tilde{w}\right), x, \tilde{w}_{0}, \tilde{w}\right)$ if $\left(\tilde{w}_{0}, \tilde{w}\right)(s) \doteq \chi_{\left[0, s_{1}[\right.}\left(w_{0}, w\right)(s)+\chi_{\left[s_{1},+\infty[\right.}\left(w_{0}, w\right)\left(s+s_{2}-s_{1}\right)$ for all $s \geq 0$. For the general case, set $\sigma=\sigma(s) \doteq \int_{0}^{s} \chi_{0,1]}\left(w_{0}\left(s^{\prime}\right)\right) \mathrm{d} s^{\prime}$ and let $s=s(\sigma)$ be the right inverse of $\sigma(\cdot)$. It is easy to see that the control $\left(\tilde{w}_{0}, \tilde{w}\right)(\sigma) \doteq\left(w_{0}, w\right)(s(\sigma))$ for all $\sigma \geq 0$ does the job. The above argument lets us immediately conclude.

In view of Proposition 3.4, the well-posedness question is significant only if $q=p$. In this case, the extended exit-time control problem is not, in general, a proper extension of the original problem, even if the weak coercivity condition (1.3) for $q=p$ is in force, as shown by the following example. 
Example 3.5. Let $\mathcal{T}=\{(0,1),(1 / 2,0)\}, A=\mathbb{R}_{+}$, and consider the control system in $\mathbb{R}^{2}$,

$$
\left\{\begin{array}{l}
\dot{y}_{1}(t)=\alpha(t) \\
\dot{y}_{2}(t)=\left|1-y_{1}(t)\right|-y_{2}(t)
\end{array}\right.
$$

with $\left(y_{1}, y_{2}\right)(0)=\left(x_{1}, x_{2}\right)$. For a fixed parameter $\beta>0$, define the value function

$$
\mathcal{V}\left(x_{1}, x_{2}\right)=\inf _{\alpha \in \mathcal{A}\left(x_{1}, x_{2}\right)} \int_{0}^{t_{x}(\alpha)}\left[\left|y_{2}(t)\left(1-y_{2}(t)\right)\right|+\beta \alpha(t)\right] \mathrm{d} t .
$$

Notice that, in view of Remark 2.2, it is not restrictive to consider only controls $\alpha \in \mathcal{B} \cap L_{\text {loc }}^{1}\left(\mathbb{R}_{+}, \mathbb{R}_{+}\right)$. Since $y_{1}$ is increasing, in order to reach $(1 / 2,0)$, any trajectory issuing from $(0,0)$ has $y_{1}(t) \in[0,1 / 2]$ for all $t>0$ and it is not difficult to see that $y_{2}(t) \geq\left(1-\mathrm{e}^{-t}\right) / 2$ for all $t>0$. This makes $(1 / 2,0)$ not reachable. Hence the only point of the target that is (asymptotically) reachable is $(0,1)$, using the control $\alpha \equiv 0$. Therefore $\mathcal{V}(0,0)=\int_{0}^{+\infty}\left(1-\mathrm{e}^{-t}\right) \mathrm{e}^{-t} \mathrm{~d} t=\frac{3}{2}$ for any choice of $\beta$.

Let us now consider the extended system

$$
\left\{\begin{array}{l}
\dot{\xi}_{1}(s)=w(s) \\
\dot{\xi}_{2}(s)=\left(\left|1-\xi_{1}(s)\right|-\xi_{2}(s)\right) w_{0}(s) \quad\left(\xi_{1}, \xi_{2}\right)(0)=\left(x_{1}, x_{2}\right)
\end{array}\right.
$$

with $\left(w_{0}, w\right) \in \Gamma$ and

$$
V\left(x_{1}, x_{2}\right)=\inf _{\left(w_{0}, w\right) \in \Gamma\left(x_{1}, x_{2}\right)} \int_{0}^{\sigma_{\left(x_{1}, x_{2}\right)}\left(w_{0}, w\right)}\left[\left|\xi_{2}(s)\left(1-\xi_{2}(s)\right)\right| w_{0}(s)+\beta w(s)\right] \mathrm{d} s,
$$

where $\Gamma\left(x_{1}, x_{2}\right)$ is given in $(3.8)$ and $\sigma_{\left(x_{1}, x_{2}\right)}\left(w_{0}, w\right)$ is defined as in $(3.7)$. In this case $(0,1)$ is reachable from the origin with the same cost as above, but now also the point $(1 / 2,0)$ is reachable using the control $\left(w_{0}, w\right)=(0,1) \chi_{[0,1 / 2]}+(1,0) \chi_{] 1 / 2,+\infty[} \in \Gamma(0,0)$ with $\sigma_{(0,0)}\left(w_{0}, w\right)=1 / 2$, and we get the cost $\beta / 2$. Therefore if $\beta<3$ one has $V(0,0)=\beta / 2<\mathcal{V}(0,0)$, while if $\beta \geq 3$ then $V(0,0)=\mathcal{V}(0,0)=\frac{3}{2}$. Notice that $\mathcal{V}$ is not continuous on $\mathcal{T}$.

In the critical case $q=p$, we have the following well-posedness result.

Theorem 3.6. Let $q=p$. If $\mathcal{V}$ is continuous on $\partial \mathcal{T}$, then $\mathcal{V} \equiv V$.

Proof of Theorem 3.6. For any $x \in \mathcal{T}^{c}$, the inequality $V(x) \leq \mathcal{V}(x)$ is obvious and, if $V(x)=+\infty$, it implies immediately $V(x)=\mathcal{V}(x)$. If $V(x)<+\infty$, let $\eta>0$. On the one hand, by the continuity of $\mathcal{V}$ on the compact set $\partial \mathcal{T}$ (where $\mathcal{V} \equiv 0$ by definition), there is some $\delta>0$ such that

$$
\mathcal{V}(\bar{x})<\eta / 3 \quad \forall \bar{x} \in \mathcal{T}^{c} \quad \text { with } \quad \mathbf{d}(\bar{x})<\delta .
$$

On the other hand, the definition of $V$ implies that

$$
\int_{0}^{\sigma_{x}\left(\tilde{w}_{0}, \tilde{w}\right)} \bar{l}\left(\tilde{\xi}_{x}, \tilde{w}_{0}, \tilde{w}\right)(s) \mathrm{d} s<V(x)+\eta / 3, \quad \mathbf{d}\left(\tilde{\xi}_{x}(\bar{s})\right)<\delta / 2
$$

for some control $\left(\tilde{w}_{0}, \tilde{w}\right) \in \Gamma(x)$ and some $\bar{s}<\sigma_{x}\left(\tilde{w}_{0}, \tilde{w}\right)$, if $\tilde{\xi}_{x}(\cdot) \doteq \xi_{x}\left(\cdot, \tilde{w}_{0}, \tilde{w}\right)$. For any $n \in \mathbb{N}$, define now

$$
w_{n} \doteq \frac{n}{n+1} \tilde{w} \chi_{[0, \bar{s}[}, \quad w_{0 n} \doteq\left(1-\left|w_{n}\right|^{q}\right)^{1 / q} .
$$

Such controls belong to $\Gamma^{+}$, the trajectories $\xi_{x}^{n}(\cdot) \doteq \xi_{x}\left(\cdot, w_{0_{n}}, w_{n}\right)$ tend uniformly to $\tilde{\xi}_{x}(\cdot)$ in $[0, \bar{s}]$ (see (A.1) and (A.3) in the Appendix) so that $\exists \bar{s}_{n} \leq \bar{s}$ such that by the regularity of $\bar{l}$ and the Dominated Convergence Theorem

$$
\int_{0}^{\bar{s}_{n}} \bar{l}\left(\xi_{x}^{n}, w_{0 n}, w_{n}\right)(s) \mathrm{d} s<V(x)+2 \eta / 3, \quad \mathbf{d}\left(\xi_{x}^{n}\left(\bar{s}_{n}\right)\right)<\delta
$$


for a sufficiently large $n$. Following the same construction of Proposition 3.1, let us set $t_{n}(s) \doteq \int_{0}^{s} w_{0}^{q}(\sigma) \mathrm{d} \sigma$ and let us denote by $s_{n}(t)$ its inverse. Let $t_{n}\left(\bar{s}_{n}\right) \doteq \bar{t}_{n}, \alpha_{n}(\cdot) \doteq \frac{w_{n}\left(s_{n}(\cdot)\right)}{w_{0 n}\left(s_{n}(\cdot)\right)}$, and $y_{x}\left(\bar{t}_{n}, \alpha_{n}\right) \doteq x_{n}$. Hence $\alpha_{n} \in$ $\mathcal{A} \cap L^{q}\left(\left[0, \bar{t}_{n}\right], A\right)$ and

$$
\int_{0}^{\bar{t}_{n}} l\left(y_{x}\left(t, \alpha_{n}\right), \alpha_{n}(t)\right) \mathrm{d} t<V(x)+2 \eta / 3, \quad \mathbf{d}\left(x_{n}\right)<\delta .
$$

Let now $\bar{\alpha}_{n} \in \mathcal{A}\left(x_{n}\right)$ be a control such that $\int_{0}^{t_{x_{n}}\left(\bar{\alpha}_{n}\right)} l\left(y_{x_{n}}\left(t, \bar{\alpha}_{n}\right), \bar{\alpha}_{n}(t)\right) \mathrm{d} t \leq \eta / 3$, which exists in view of (3.11). Then, the control $\left.\alpha(t)=\alpha_{n}(t) \chi_{\left[0, \bar{t}_{n}\right]}(t)+\bar{\alpha}_{n}\left(t-\bar{t}_{n}\right) \chi_{]} \bar{t}_{n},+\infty\right](t)$ belongs to $\mathcal{A}(x)$ and

$$
\mathcal{V}(x) \leq \int_{0}^{t_{x}(\alpha)} l\left(y_{x}(t, \alpha), \alpha(t)\right) \mathrm{d} t \leq V(x)+\eta .
$$

By the arbitrariness of $\eta>0$, the proof is concluded.

\subsection{Well posedness of $V^{m}$}

Differently from $V$ and $\mathcal{V}, V^{m}$ and $\mathcal{V}^{m}$ do not coincide in general, even if $\mathcal{V}^{m}$ is continuous in some neighborhood of $\mathcal{T}$, as shown in the following example.

Example 3.7. Let $\mathcal{T}=\left\{\left(x_{1}, x_{2}\right): x_{1}=10,1 \leq x_{2} \leq 3\right\}$ and consider the bi-dimensional system

$$
\left\{\begin{array}{l}
\dot{y}_{1}(t)=\alpha(t) \\
\dot{y}_{2}(t)=f\left(y_{1}, y_{2}\right)
\end{array}\right.
$$

where $\left(y_{1}, y_{2}\right)(0)=\left(x_{1}, x_{2}\right), \alpha(t) \in \mathbb{R}$ and $f\left(x_{1}, x_{2}\right)$ is any Lipschitz continuous nonnegative function, positive for $x_{1}>0$, extension to $\left\{\left(x_{1}, x_{2}\right): x_{2}>-1\right\}$ of the following Lipschitz continuous function defined in $D=$ $\left\{\left(x_{1}, x_{2}\right): 3 \leq x_{1} \leq 12,-1 \leq x_{2} \leq 4\right\} \cup\left\{\left(0, x_{2}\right): x_{2} \geq 0\right\}$

$$
g\left(x_{1}, x_{2}\right)=\left\{\begin{array}{l}
x_{2} \quad \text { if } x_{1}=0 \\
1 \quad \text { if }\left(3 \leq x_{1} \leq 12\right) \wedge\left(-1 \leq x_{2} \leq 1\right) \\
-1 \quad \text { if }\left(3 \leq x_{1} \leq 12\right) \wedge\left(3 \leq x_{2} \leq 4\right) \\
2-x_{2} \quad \text { if }\left(3 \leq x_{1} \leq 12\right) \wedge\left(1 \leq x_{2} \leq 3\right) .
\end{array}\right.
$$

For $x=\left(x_{1}, x_{2}\right)$ the cost is given by

$$
\mathcal{J}(T, x, \alpha)=\int_{0}^{T}\left|y_{2}(t)\left(10-y_{1}(t)\right)\right|+|\alpha(t)| \mathrm{d} t .
$$

Notice that by Remark 2.2 we can minimize over the subset of controls $\alpha \in \mathcal{B} \cap L_{\mathrm{loc}}^{1}\left(\mathbb{R}_{+}, \mathbb{R}\right) \subset \mathcal{A}$. We have

$$
V(x)=\mathcal{V}(x)=\left|10-x_{1}\right| \quad \forall x \in D .
$$

In fact, for every $x=\left(x_{1}, x_{2}\right) \in D$ let us prove that, setting $\alpha_{n}(t) \doteq\left(10-x_{1}\right) n \chi_{\left[0, \frac{1}{n}\right]}(t),\left(\alpha_{n}\right)_{n}$ is a minimizing sequence of controls. If $\left|x_{2}-2\right| \leq 1$ we have $y_{x}\left(n^{-1}, \alpha_{n}\right) \in \mathcal{T}$ since the second component of the trajectory issuing from $x$ is monotone and such that $\lim _{t \rightarrow 0^{+}} y_{2}\left(t, \alpha_{n}\right)=x_{2}$. Hence the Dominated Convergence Theorem yields $\lim _{n \rightarrow+\infty} \mathcal{J}\left(n^{-1}, x, \alpha_{n}\right)=\left|10-x_{1}\right|$. Otherwise, for $x=\left(x_{1}, x_{2}\right) \in D$ with $\left|x_{2}-2\right|>1$, it is $y_{x}\left(n^{-1}, \alpha_{n}\right)=$ $\left(10, x_{2}+\operatorname{sgn}\left(\left|x_{2}-2\right|-1\right) n^{-1}\right)$. For $n$ large, set $t_{n} \doteq\left|x_{2}-2\right|-1-n^{-1}>0$. The null control running for $n^{-1} \leq t \leq t_{n}+n^{-1}$ leads the trajectory to the target. Moreover since $y_{1}(t) \equiv 10$ for $t>n^{-1}$ we have $\mathcal{J}\left(n^{-1}+t_{n}, x, \alpha_{n}\right)=\mathcal{J}\left(n^{-1}, x, \alpha_{n}\right)$ and we can conclude as in the previous case. Finally $\mathcal{V}(x)=\left|10-x_{1}\right|$, since for any $\alpha \in \mathcal{A}(x)$ it is easy to see that one has $\mathcal{V}(x) \geq \int_{0}^{t_{x}(\alpha)}|\alpha(t)| \mathrm{d} t \geq\left|10-x_{1}\right|$.

Let us now consider the associated extended system, given by

$$
\left\{\begin{array}{l}
\dot{\xi}_{1}(s)=w(s) \\
\dot{\xi}_{2}(s)=f\left(\xi_{1}, \xi_{2}\right) w_{0}(s)
\end{array}\right.
$$


and the extended cost

$$
J\left(S, x, w_{0}, w\right)=\int_{0}^{S}\left|\xi_{2}(s)\left(10-\xi_{1}(s)\right)\right| w_{0}(s)+|w(s)| \mathrm{d} s .
$$

Since $\mathcal{V}$ is continuous on $\partial \mathcal{T}, V \equiv \mathcal{V}$ owing to Theorem 3.6. However, at any point $\left(x_{1}, 0\right)$ with $x_{1}>0$, we get

$$
V^{m}\left(x_{1}, 0\right)=\left\{\begin{array}{l}
x_{1}, \quad \text { for } 0<x_{1}<5 \\
10-x_{1}, \quad \text { for } 5 \leq x_{1} \leq 10
\end{array}\right.
$$

The optimal cost to reach the origin is, indeed, $J\left(\infty,\left(x_{1}, 0\right), \tilde{w}_{0}, \tilde{w}\right)=x_{1}$, obtained by implementing the control $\left(\tilde{w}_{0}, \tilde{w}\right)(s)=(0,-1) \chi_{\left[0, x_{1}\right]}(s)+(1,0) \chi_{\left[x_{1},+\infty[\right.}$.

So $V^{m}\left(x_{1}, 0\right)<V\left(x_{1}, 0\right)=\mathcal{V}\left(x_{1}, 0\right)$ for all $0<x_{1}<5$, being more convenient, if allowed, to reach the origin, which belongs to $\mathcal{Z}$ but not to the target. Actually, this can be done only using an impulsive control, where $w_{0}=0$ on some interval, whose corresponding extended trajectory is not limit of trajectories of the original system with finite cost. It is now easy to see that $\mathcal{V}^{m} \equiv \mathcal{V} \equiv V$ in the interior of $D$, so that $\mathcal{V}^{m}$ is continuous in it, but $V^{m}\left(x_{1}, 0\right)<\mathcal{V}^{m}\left(x_{1}, 0\right)$ for all $3<x_{1}<5$. For any control $\alpha \in \mathcal{B} \cap L_{\text {loc }}^{1}\left(\mathbb{R}_{+}, \mathbb{R}\right) \backslash \mathcal{A}(x)$ with $x \in \stackrel{\circ}{D}$, we have indeed that there exists some $\bar{c}>0$ such that $\mathcal{J}(+\infty, x, \alpha) \geq \bar{c} \int_{0}^{+\infty}\left|y_{2}(t)\right| \mathrm{d} t=+\infty$, since $y_{2}(\cdot)$ is increasing for $t \geq 0$. Therefore the infimum over $\mathcal{A}$ has to be reached on $\mathcal{A}(x)$ and $\mathcal{V}^{m}(x)=\mathcal{V}(x)$.

Notice that the lagrangian of Example 3.7 is weakly coercive. Coercivity for $q>p$ is though sufficient to have $\mathcal{V}^{m}=V^{m}$. In fact we obtain the following general result.

Proposition 3.8. If either the coercivity condition (1.3) or the target-weighted coercivity hypothesis (1.4) for $q>p$ holds, then $V^{m} \equiv \mathcal{V}^{m}$.

Proof. Arguing as in the proof of Proposition 3.4, the cost of any control $\left(w_{0}, w\right) \in \Gamma \backslash \Gamma^{+}$such that $\int_{0}^{+\infty} w_{0}(s) \mathrm{d} s>0$, is greater than or equal to the cost associated to a suitable control $\left(\tilde{w}_{0}, \tilde{w}\right) \in \Gamma^{+}$(corresponding to an original control $\alpha$, in view of Prop. 3.1). Hence we only have to show that any control $(0, w) \in \Gamma$ cannot be optimal. As observed in the proof of Proposition 3.4, for any $x \in \mathcal{T}^{c},(0, w) \in \Gamma \backslash \Gamma(x)$. Hence, since $w_{0}^{q}+|w|^{q}=1, \sigma_{x}(0, w)=\int_{0}^{\sigma_{x}(0, w)}|w(s)|^{q} \mathrm{~d} s=+\infty$ and assuming either (1.3) or (1.4), one obtains $J(+\infty, x, 0, w)=+\infty$.

Another situation where $\mathcal{V}^{m} \equiv V^{m}$, is the degenerate case $l(x, a) \equiv l(x)$. Precisely, we prove what follows.

Proposition 3.9. Assume $l(x, a) \equiv l(x)$. Then $V^{m} \equiv 0$ and, if

$$
l(x) \leq \bar{M}\left(1+|x|^{r}\right) \quad \forall(x, a) \in \mathbb{R}^{n} \times \mathcal{T}
$$

for some $r, \bar{M}>0$, we also have $\mathcal{V}^{m} \equiv 0$.

Proof. For any $x \in \mathcal{T}^{c}, V^{m}(x)=0$ since $\bar{l}\left(x, w_{0}, w\right)=l(x) w_{0}^{p}$ and $J(+\infty, x, 0, w)=0$ for any control $(0, w) \in \Gamma$ ( $p$ the same as in (H0)). Let $0<T_{n} \leq 1$ for $n \in \mathbb{N}$ be a positive, decreasing sequence converging to zero. For any $n \in \mathbb{N}$, let $\alpha_{n} \in \mathcal{B} \cap L_{\mathrm{loc}}^{p}\left(\left[0, T_{n}[, A)\right.\right.$ be a control such that

$$
k_{n}(t) \doteq \int_{0}^{t}\left|\alpha_{n}(t)\right|^{p} \mathrm{~d} t=\log \left(\left|\log \left(T_{n}-t\right)\right|\right) \quad \forall t \in\left[0, T_{n}[,\right.
$$

which always exists, since $A$ is a cone. Therefore $T_{\alpha_{n}}=T_{n}$ and, setting $y_{x}^{n}(\cdot) \doteq y_{x}\left(\cdot, \alpha_{n}\right)$, by $(2.5)$ it follows that

$$
\left|y_{x}^{n}(t)\right| \leq\left[|x|+M\left(t+k_{n}(t)\right)\right] \mathrm{e}^{M\left(t+k_{n}(t)\right)} \quad t \in\left[0, T_{n}[.\right.
$$

Hence we get

$$
\int_{0}^{T_{n}} l\left(y_{x}^{n}(t)\right) \mathrm{d} t \leq \bar{M}\left(T_{n}+\int_{0}^{T_{n}}\left|y_{x}^{n}(t)\right|^{r} \mathrm{~d} t\right) \leq \bar{C}\left(T_{n}+\int_{0}^{T_{n}} k_{n}^{r}(t) \mathrm{e}^{M r k_{n}(t)} \mathrm{d} t\right)
$$


for some $\bar{C}>0$. The previous estimates imply that

$$
\int_{0}^{T_{n}} l\left(y_{x}^{n}(t)\right) \mathrm{d} t \leq \bar{C}\left(T_{n}+\int_{0}^{T_{n}} \log ^{r}\left(\left|\log \left(T_{n}-t\right)\right|\right)\left|\log \left(T_{n}-t\right)\right|^{M r} \mathrm{~d} t\right)
$$

where the last expression tends to zero as $n$ tends to $+\infty$. Indeed, by the substitution $s=T_{n}-t$, we have

$$
\int_{0}^{T_{n}} \log ^{r}\left(\left|\log \left(T_{n}-t\right)\right|\right)\left|\log \left(T_{n}-t\right)\right|^{M r} \mathrm{~d} t=\int_{0}^{T_{n}} \log ^{r}(|\log (s)|)|\log (s)|^{M r} \mathrm{~d} s,
$$

which tends to zero as $T_{n} \rightarrow 0^{+}$, since $\log ^{r}(|\log (s)|)|\log (s)|^{M r}$ is an integrable function at $0^{+}$.

In the following example, $l$ is as in (3.16) and the asymptotic value function $\mathcal{V}(x)$ is equal to zero. The controls of any minimizing sequence use infinite energy and their blow-up times converge to 0 .

Example 3.10. Let us consider the scalar system

$$
\dot{y}(t)=-\alpha(t) y(t)
$$

where $y(0)=x$ and $\alpha(t) \in \mathbb{R}_{+}$. The target is $\mathcal{T}=\{0\}$ and the payoff is given by

$$
\mathcal{J}(T, x, \alpha)=\int_{0}^{T}(1+|y(t)|) \mathrm{d} t .
$$

For any $x \neq 0$ and $\alpha \in \mathcal{A}, y_{x}(t, \alpha)=x \mathrm{e}^{-k(t)}$, where $k(t)=\int_{0}^{t} \alpha\left(t^{\prime}\right) \mathrm{d} t^{\prime}$ for $t \geq 0$. For the same choice of controls $\alpha_{n}$ used in the proof of Proposition 3.9 for $p=1$, one has $\lim _{t \rightarrow T_{n}^{-}} y_{x}\left(t, \alpha_{n}\right)=0$ and $\lim _{n} J\left(T_{n}, x, \alpha_{n}\right)=0$, so that $\mathcal{V}(x)=0$. Notice that for every $x \in \mathcal{T}^{c}$, any admissible control $\alpha \in \mathcal{A}(x)$ must have $\int_{0}^{t_{x}(\alpha)}|\alpha(t)| \mathrm{d} t=+\infty$. Moreover, any minimizing control sequence $\left(\alpha_{n}\right)_{n}$ has finite blow-up times $T_{\alpha_{n}}$ converging to zero.

At this point one wonders if in the case $l(x, a) \equiv l(x)$ the value functions $\mathcal{V}$ and $V$ are themselves identically zero. The answer is given in the next example where we have $\mathcal{V}, V \neq 0$ and an asymptotically reachable target.

Example 3.11. Let us consider the scalar system

$$
\dot{y}(t)=-y(t)+\alpha(t),
$$

where $y(0)=x$ and $\alpha(t) \in \mathbb{R}_{+}$. The target is $\mathcal{T}=\{0\}$ and the payoff is given by

$$
\mathcal{J}(T, x, \alpha)=\int_{0}^{T}|y(t)| \mathrm{d} t .
$$

Hence $p=q=1$, the extended system and payoff are given, respectively, by

$$
\dot{\xi}(s)=-\xi(s) w_{0}(s)+w(s), \quad J\left(S, x, w_{0}, w\right)=\int_{0}^{S}|\xi(r)| w_{0}(r) \mathrm{d} r
$$

and we get

$$
V(x)=\mathcal{V}(x)= \begin{cases}x & \text { if } x \geq 0 \\ 0 & \text { if } x<0\end{cases}
$$

Indeed, if $x \leq 0$ it is enough to choose the minimizing sequence of controls, $\alpha_{n}(t) \doteq n \chi_{[0, \log (1-x / n)]}(t)$. On the other hand, for every $x>0$, the optimal control is $\alpha \equiv 0$. Notice that for $x>0$, any control $\alpha \in \mathcal{A}(x)$ gives rise to a cost not smaller that $x$. Indeed, first of all, if for some $T_{\alpha}<+\infty$ one had $\int_{0}^{T_{\alpha}}|\alpha(s)| \mathrm{d} s=+\infty$, then $y_{x}(t, \alpha) \rightarrow+\infty$ as $t \rightarrow T_{\alpha}$ and hence $\alpha \notin \mathcal{A}(x)$. Therefore it is easy to see thet for any $\alpha \in \mathcal{A}(x), t_{x}(\alpha)=+\infty$ and $y_{x}(t, \alpha)=\mathrm{e}^{-t}\left(x+\int_{0}^{t} \mathrm{e}^{s} \alpha(s) \mathrm{d} s\right) \geq x \mathrm{e}^{-t}$ so that

$$
\mathcal{J}(+\infty, x, \alpha) \geq \int_{0}^{+\infty} x \mathrm{e}^{-s} \mathrm{~d} s=x .
$$




\section{Optimality PRinciples AND Uniqueness}

This section is devoted to introduce sufficient conditions in order to characterize $\mathcal{V}$ as unique non negative solution of a Hamilton-Jacobi equation with suitable boundary conditions. We first show that, under the following assumption

$$
\forall x \in \mathcal{T}^{c}: \quad\left|f^{\infty}(x, a)\right|+l^{\infty}(x, a) \neq 0 \quad \forall a \in A \backslash\{0\},
$$

the boundary value problem, in short (BVP), associated to the exit-time value functions defined in the previous sections can be reformulated in terms of the continuous Hamiltonian $H$ defined in (4.5) below. Furthermore we obtain the optimality principles in Proposition 4.5 and the uniqueness Theorems 4.6, 4.7 by refining analogous results of [12], proved there under hypothesis (1.3). In view of the optimality principles, the question of uniqueness is reduced here, for both the cases $q>p$ and $q=p$, to the control theoretical question of whether $V$ coincides with $V^{m}$. In order to unify the exposition, we use the extended value functions even when they are equal to the original value functions. We recall that, owing to Propositions 3.4 and 3.8 , this happens for instance if either the coercivity hypothesis (1.3) or the target-weighted coercivity condition (1.4) for $q>p$ holds.

Remark 4.1. Both conditions (1.3) and (1.4) for $q \geq p$ imply (4.1). Nevertheless, hypothesis (4.1) may be verified even in very degenerate, cheap control problems, where $l(x, a) \equiv l(x)$, so that $l^{\infty}(x, a) \equiv 0$. In this case, if $f(x, a)=f_{0}(x)+\sum_{i=1}^{m} f_{i}(x) a_{i}$, then (4.1) is equivalent to assume $\sum_{i=1}^{m} f_{i}(x) a_{i} \neq 0$ for all $(x, a) \in \mathcal{T}^{c} \times(A \backslash\{0\})$.

In order to apply the results of [12], throughout this section we assume that

$$
\text { for any } R>0 \text {, there exists } \bar{L}_{R}>0 \text { such that } \omega(r, R)=\bar{L}_{R} r \text {, }
$$

where $\omega$ is the modulus of continuity of $l$ in (H0). In fact, the sublinear growth of $l$ assumed in [12] can be removed as in [9].

\subsection{The boundary value problem}

For any $(x, p) \in \mathbb{R}^{n} \times \mathbb{R}^{n}$, define the Hamiltonian

$$
\mathcal{H}(x, p) \doteq \sup _{a \in A}\{-\langle p, f(x, a)\rangle-l(x, a)\} .
$$

\section{Definition 4.2 .}

(BVP) Any function $v: \mathbb{R}^{n} \backslash \stackrel{\circ}{\mathcal{T}} \rightarrow \mathbb{R} \cup\{+\infty\}$ verifying $v_{*}(x) \geq 0$ on $\partial \mathcal{T}$ and such that $v_{*}$ is a viscosity supersolution of

$$
\mathcal{H}(x, D v(x))=0
$$

in $\mathbb{R}^{n} \backslash \mathcal{T}$, is called a supersolution to (BVP). Any pair $(v, \Omega)$ where $\Omega \supset \mathcal{T}$ is an open set and $v: \Omega \backslash \mathcal{T} \rightarrow \mathbb{R}$ is a locally bounded function verifying $v^{*}(x) \leq 0$ on $\partial \mathcal{T}$ and such that $v^{*}$ is a viscosity subsolution of (4.3) in $\Omega \backslash \mathcal{T}$, is called a subsolution to (BVP) (in $\Omega$ ).

Any pair $(v, \Omega)$, where $v: \mathbb{R}^{n} \backslash \stackrel{\circ}{\mathcal{T}} \rightarrow \mathbb{R} \cup\{+\infty\}$ and $\Omega$ is an open set, $\Omega \supset \mathcal{T}$, is called a solution to (BVP) (in $\Omega$ ) if $v$ is supersolution and $(v, \Omega)$ is a subsolution to (BVP).

For precise definitions and more comments on (BVP), we refer to [12]. Here we just point out that the exit-time value functions do not satisfy in general the boundary condition

$$
\lim _{x \rightarrow \bar{x}} v(x)=+\infty \quad \forall \bar{x} \in \partial \operatorname{Dom}(v) .
$$

If no coercivity is assumed, $\mathcal{H}$ may be discontinuous and equal to $+\infty$ in some points. In this case, following the viscosity theory, in (BVP) one has to consider the upper and lower semi-continuous envelope of $\mathcal{H}, \mathcal{H}^{*}$ and $\mathcal{H}_{*}$, respectively. In particular $v$ is a subsolution if $v^{*}$ is a subsolution to $\mathcal{H}_{*}(x, D v)=0$ while $v$ is a 
supersolution if $v_{*}$ is a supersolution to $\mathcal{H}^{*}(x, D v)=0$. However, in view of Proposition 4.3 below, all the results in the sequel hold with $\mathcal{H}$ replaced for any $(x, p) \in \mathbb{R}^{n} \times \mathbb{R}^{n}$ by the following continuous extended Hamiltonian $H$,

$$
H(x, p) \doteq \max _{\left(w_{0}, w\right) \in S(A)}\left\{-\left\langle p, \bar{f}\left(x, w_{0}, w\right)\right\rangle-\bar{l}\left(x, w_{0}, w\right)\right\}
$$

where $\bar{f}, \bar{l}, S(A)$ are defined as in (3.1). Actually, considering $H$ turns out to be useful even if $\mathcal{H}$ is coercive, since it allows to obtain optimality principles for dynamics verifying $|f(x, a)| \leq M\left(1+|a|^{p}\right)(1+|x|)$ instead of the more restrictive hypothesis $|f(x, a)| \leq M\left(1+|a|^{p}+|x|\right)$, assumed in most of the literature (see e.g. $[9,18]$ ). An analogous remark holds for $l$.

Proposition 4.3. Let $u: \mathbb{R}^{n} \backslash \stackrel{\circ}{\mathcal{T}} \rightarrow \mathbb{R} \cup\{+\infty\}$.

(i) $u$ is a subsolution of $\mathcal{H}(x, D u(x))=0$ at some $x$ iff it is a subsolution of $H(x, D u(x))=0$ at $x$;

(ii) if $u$ is a supersolution of $\mathcal{H}(x, D u(x))=0$ at some $x$, then it is a supersolution of $H(x, D u(x))=0$ at $x$. When (4.1) holds, also the converse implication is true.

Proof. In Theorem 2.1, [12] the hypothesis (1.3) is assumed. From the proof, however, it is immediate to see that (1.3) is not necessary to prove statement (i) and the first implication in (ii). For the second implication in (ii), let us assume that $u$ is a viscosity supersolution to $H(x, D u)=0$ at $x$. If $u(x)=+\infty$ the thesis is trivial. Let $u(x)<+\infty$ and let $\varphi \in \mathcal{C}^{1}\left(\mathbb{R}^{n}\right)$ be such that $u-\varphi$ has a local minimum at $x$. Since $H$ is continuous, one has $H=H^{*}$ and $H(x, D \varphi(x)) \geq 0$. Let $\left(\bar{w}_{0}, \bar{w}\right) \in S(A)$ be such that

$$
\left\langle-D \varphi(x), \bar{f}\left(x, \bar{w}_{0}, \bar{w}\right)\right\rangle-\bar{l}\left(x, \bar{w}_{0}, \bar{w}\right)=\max _{\left(w_{0}, w\right) \in S(A)}\left\{-\left\langle p, \bar{f}\left(x, w_{0}, w\right)\right\rangle-\bar{l}\left(x, w_{0}, w\right)\right\} \geq 0 .
$$

If $\bar{w}_{0}>0$ by setting $a=\frac{\bar{w}}{\bar{w}_{0}}$ one can easily prove that $\mathcal{H}^{*}(x, D \varphi(x)) \geq 0$. Let $\bar{w}_{0}=0$. If $\bar{l}(x, 0, \bar{w})=l^{\infty}(x, \bar{w})>0$, one has $\bar{f}(x, 0, \bar{w})=f^{\infty}(x, \bar{w}) \neq 0$ since otherwise (4.6) would be false, while in case $\bar{l}(x, 0, \bar{w})=0$, the condition $\bar{f}(x, 0, \bar{w}) \neq 0$ is guaranteed by (4.1). In both situations, there exists $\mu(x)>0$ such that $|f(x, 0, \bar{w})|^{2} \geq \mu(x)>0$. Let $\left(h_{n}\right)_{n}$ be a sequence of positive numbers with $h_{n} \leq 1$ and set $p_{n} \doteq D \varphi(x)-h_{n} \bar{f}(x, 0, \bar{w})$. One has

$$
\sup _{\left(w_{0}, w\right) \in S(A)}\left\{-\left\langle p_{n}, \bar{f}\left(x, w_{0}, w\right)\right\rangle-\bar{l}\left(x, w_{0}, w\right)\right\} \geq h_{n} \mu(x),
$$

so that there exists a sequence $\left(w_{0_{n}}, w_{n}\right)$ with $w_{0_{n}}>0$ such that

$$
-\left\langle p_{n}, f\left(x, a_{n}\right)\right\rangle-l\left(x, a_{n}\right) \geq \frac{h_{n} \mu(x)}{2 w_{0_{n}}^{q}}>0
$$

where we have set $a_{n}=\frac{w_{n}}{w_{0_{n}}}$. Hence $\sup _{a \in A}\left\{-\left\langle p_{n}, f(x, a)\right\rangle-l(x, a)\right\}>0$ which implies $\mathcal{H}^{*}(x, D \varphi(x)) \geq 0$.

\section{Proposition 4.4.}

(i) Let $W \in\left\{\mathcal{V}, V, V^{m}\right\}$. If $W$ is locally bounded in $\operatorname{Dom}(W)$, $\operatorname{Dom}(W)$ is open and $W^{*} \leq 0$ on $\partial \mathcal{T}$, then $(W, \operatorname{Dom}(W))$ is a subsolution to (BVP).

(ii) $\mathcal{V}$ is a nonnegative supersolution to (BVP). If (4.1) holds, then $V$ and $V^{m}$ are nonnegative supersolutions to (BVP) too.

We omit the proof, since it is analogous to that of Proposition 3.2 in [12].

\subsection{Maximal and minimal solutions and uniqueness}

Let us briefly introduce the relaxed extended minimal value function, $V^{m, r}$, obtained by taking the infimum over relaxed extended controls, $\mu(\cdot) \in \Gamma^{r} \doteq L^{\infty}\left(\mathbb{R}_{+}, \mathcal{P}(\overline{B(0,1)} \cap A)\right)$. As usual, $A^{r} \doteq \mathcal{P}(\overline{B(0,1)} \cap A)$ is theset of 
Radon probability measures on the compact set $\overline{B(0,1)} \cap A$ endowed with the weak ${ }^{*}$ topology and we consider $\psi \in\{\bar{f}, \bar{l}\}$ extended to $\mathbb{R}^{n} \times A^{r}$ by setting

$$
\psi^{r}(x, \mu) \doteq \int_{\overline{B(0,1)} \cap A} \psi\left(x,\left(1-|w|^{q}\right)^{1 / q}, w\right) \mathrm{d} \mu \quad \forall \mu \in A^{r} .
$$

For any $x \in \mathcal{T}^{c}$ and $\mu \in \Gamma^{r}, \xi_{x}^{r}(s, \mu)$ denotes the relaxed trajectory, solution of

$$
\dot{\xi}^{r}=\bar{f}^{r}\left(\xi^{r}, \mu\right) \quad \text { for } s>0, \quad \xi^{r}(0)=x
$$

and we set $\sigma_{x}^{r}(\mu) \doteq \inf \left\{s>0: \xi_{x}^{r}(s, \mu) \in \mathcal{T}\right\} \quad(\leq+\infty)$. Finally, we define

$$
V^{m, r}(x) \doteq \inf _{\mu \in \Gamma^{r}} \int_{0}^{\sigma_{x}^{r}(\mu)} \bar{l}^{r}\left(\xi_{x}^{r}(s, \mu), \mu(s)\right) \mathrm{d} s .
$$

\section{Proposition 4.5.}

(i) Assume (4.1). Then $V^{m} \leq u$ for any nonnegative and continuous supersolution $u$ to (BVP), $V^{m, r}$ is l.s.c and it is the minimal nonnegative supersolution to (BVP).

(ii) If $V$ is continuous on $\partial \mathcal{T}$, then $(V, \operatorname{Dom}(V))$ is the maximal subsolution to (BVP) among the pairs $(v, \operatorname{Dom}(V))^{2}$.

Proof. We only sketch the proof of (i), where some changes with respect to the proof of Theorem 4.3 in [12] are needed and omit that of (ii), which is the same as in [12]. Actually, we just consider the case where $u$ is continuous (for $u$ just l.s.c. the rearrangements are obvious). In view of Theorem 4.1 in [12], any nonnegative continuous viscosity supersolution $u$ to (BVP) satisfies $\forall x \in \mathcal{T}^{c}$ the following upper optimality principle ${ }^{3}$ :

$$
u(x)=\inf _{\left(w_{0}, w\right) \in \mathcal{B}\left(\mathbb{R}_{+}, S(A)\right)} \sup _{0 \leq s<\sigma_{x}\left(w_{0}, w\right)}\left[J\left(s, x, w_{0}, w\right)+u\left(\xi_{x}\left(s, w_{0}, w\right)\right)\right],
$$

which implies that

$$
u(x) \geq \inf _{\left(w_{0}, w\right) \in \mathcal{B}\left(\mathbb{R}_{+}, S(A)\right)} \limsup _{s \rightarrow \sigma_{x}\left(w_{0}, w\right)}\left[J\left(s, x, w_{0}, w\right)+u\left(\xi_{x}\left(s, w_{0}, w\right)\right)\right] .
$$

Since $u$ is continuous and $u \geq 0$ in $\mathcal{T}^{c}$, by (4.8) it follows that

$$
\limsup _{s \rightarrow \sigma_{x}\left(w_{0}, w\right)}\left[J\left(s, x, w_{0}, w\right)+u\left(\xi_{x}\left(s, w_{0}, w\right)\right)\right] \geq J\left(\sigma_{x}\left(w_{0}, w\right), x, w_{0}, w\right)
$$

for all $\left(w_{0}, w\right) \in \mathcal{B}\left(\mathbb{R}_{+}, S(A)\right)$. The last two estimates yield $u(x) \geq V^{m}(x)$.

Let us now state the following uniqueness result, whose proof follows from Theorem 4.7 below.

Theorem 4.6. Assume (4.1).

(i) If $V \equiv V^{m}$ and $\mathcal{V}$ is continuous in $\operatorname{Dom}(\mathcal{V})$ and satisfies the boundary condition (4.4), then $\mathcal{V}\left(\equiv V \equiv V^{m}\right)$ is the unique nonnegative viscosity solution to (BVP) among the pairs $(v, \Omega)$, where $v$ is continuous in $\Omega$ and $\lim _{x \rightarrow \bar{x}} v(x)=+\infty \quad \forall \bar{x} \in \partial \Omega$.

(ii) If $V \equiv V^{m, r}$ and $\mathcal{V}$ is continuous on $\partial \mathcal{T}$, then $(\mathcal{V}, \operatorname{Dom}(\mathcal{V}))\left(\right.$ where $\left.\mathcal{V} \equiv V \equiv V^{m, r}\right)$ is the unique nonnegative viscosity solution to (BVP) among the pairs $(v, \Omega)$, where $v$ satisfies (4.4). Moreover, $\mathcal{V}$ is continuous ${ }^{4}$.

\footnotetext{
${ }^{2}$ We recall that $\operatorname{Dom}(V)$ is an open set, $V$ is locally bounded and upper semicontinuous in view of Proposition 2.6 (reformulated for the extended problem).

${ }^{3}$ Differently from [12], where the infimum was taken over the subset of controls of $\mathcal{B}\left(\mathbb{R}_{+}, S(A)\right)$, such that $\int_{0}^{+\infty} w_{0}^{q}(s) \mathrm{d} s=+\infty$, we drop here the integral constraint. The two infimums coincide if (1.3) is assumed, as done in [12] (see Rems. 2.2, 3.3).

${ }^{4}$ Since $V^{m, r}$ is lsc and $\mathcal{V}$ is usc, when $\mathcal{V} \equiv V^{m, r}(4.4)$ is trivially satisfied.
} 
Notice that, unless either (1.3) or (1.4) for $q>p$ is in force, in view of the results in Section 3.1 condition $V \equiv V^{m}$ is stronger than the hypothesis $\mathcal{V} \equiv \mathcal{V}^{m}$ assumed in an analogous uniqueness theorem obtained in [16], for the case of bounded controls.

By a Kruzkov transform the free boundary problem (BVP) can be replaced by another boundary value problem in $\mathbb{R}^{n} \backslash \mathcal{T}$, whose solution, when unique, simultaneously gives both $v$ and $\Omega \doteq \operatorname{Dom}(v)$. More precisely, we introduce the extended Hamiltonian

$$
K(x, u, p) \doteq \max _{\left(w_{0}, w\right) \in S(A)}\left\{-\left\langle p, \bar{f}\left(x, w_{0}, w\right)\right\rangle-\bar{l}\left(x, w_{0}, w\right)+\bar{l}\left(x, w_{0}, w\right) u\right\},
$$

and we consider the following boundary value problem, in short, (BVPK),

$$
\left\{\begin{array}{l}
K(x, W(x), D W(x))=0 \quad \text { in } \mathbb{R}^{n} \backslash \mathcal{T} \\
W(x)=0 \quad \text { on } \partial \mathcal{T}
\end{array}\right.
$$

where super- and subsolutions are defined analogously to Definition 4.2.

We have the following uniqueness result in $\mathbb{R}^{n}$.

Theorem 4.7. Assume (4.1).

(i) If $V \equiv V^{m}$ and $\mathcal{V}$ is continuous in $\operatorname{Dom}(\mathcal{V})$ and satisfies the boundary condition (4.4), then there is a unique nonnegative viscosity solution $\mathcal{U}$ to $(\mathrm{BVPK})$ among the continuous functions. Moreover, $\mathcal{V} \equiv \Psi^{-1}(\mathcal{U})=$ $-\log (1-\mathcal{U})$ and $\operatorname{Dom}(\mathcal{V})=\{x: \mathcal{U}(x)<1\}$.

(ii) If $V \equiv V^{m, r}$ and $\mathcal{V}$ is continuous on $\partial \mathcal{T}$, then there is a unique nonnegative viscosity solution $\mathcal{U}$ to $(\mathrm{BVP \mathcal {K }})$ which turns out to be continuous. Moreover, $\mathcal{V} \equiv \Psi^{-1}(\mathcal{U})=-\log (1-\mathcal{U})$ and $\operatorname{Dom}(\mathcal{V})=\{x: \mathcal{U}(x)<1\}$.

Proof. Let us prove (ii), the proof of (i) being analogous and actually simpler. By Proposition 4.5 and the boundary condition (4.4), for any solution $W$ to (BVPK) we get, for every $x \in \mathbb{R}^{n} \backslash \mathcal{T}$,

$$
\Psi\left(V^{m, r}\right)(x) \leq W_{*}(x) \leq W(x) \leq W^{*}(x) \leq \Psi(V)(x) .
$$

By Proposition 3.4 and Theorem 3.6 for the case $q=p, V \equiv \mathcal{V}$. Since $V \equiv V^{m, r}$, the thesis follows now easily.

For the continuity of $\mathcal{V}$ on $\partial \mathcal{T}$ we refer to Theorem 2.4 and Remark 2.5, and to Section 6 for its global continuity. In the next Section we will establish some sufficient conditions in order to have $V \equiv V^{m}$ or $V \equiv V^{m, r}$. We point out that when $V \neq V^{m}$, owing to Proposition 4.5 we can still characterize $\mathcal{V}$ as maximal subsolution of $(\mathrm{BVP})$. Alternatively, in Section 5 we will obtain $\mathcal{V}$ as limit of some penalized problems.

\subsection{Conditions to have $V \equiv V^{m}$ or $V \equiv V^{m, r}$}

\subsubsection{Conditions for $V \equiv V^{m}$}

Preliminarly, let us notice that, by definition, $V \equiv V^{m}$ if and only if for any $x \in \mathcal{T}^{c}$, one has

$$
\int_{0}^{+\infty} \bar{l}\left(\xi_{x}\left(s, w_{0}, w\right), w_{0}(s), w(s)\right) \mathrm{d} s \geq V(x) \quad \forall\left(w_{0}, w\right) \in \Gamma \backslash \Gamma(x) .
$$

Clearly, a first situation where (4.11) holds, is when $\Gamma \equiv \Gamma(x)$ for any $x \in \mathcal{T}^{c}$. For instance, we can get such an equality if the extended control system (3.3) is globally asymptotically stable, in short, GAS, w.r.t. $\partial \mathcal{T}$ in $\mathcal{T}^{c}$, which, roughly means that all extended trajectories converge to $\partial \mathcal{T}$. We recall that (3.3) is said uniformly $G A S, U G A S$, w.r.t. $\partial \mathcal{T}$ if, in addition, initial conditions in a compact set give rise to trajectories approaching the target uniformly. The literature on this subject is huge and we refer the interested reader to [1] and the references therein. Here we skip precise definitions and, limit ourselves to give a sufficient condition, in terms of a Lyapunov function, yielding that (3.3) is UGAS w.r.t. $\partial \mathcal{T}$. 
(SC1) There exists a function $U: \mathbb{R}^{n} \backslash \stackrel{\circ}{\mathcal{T}} \rightarrow \mathbb{R}_{+}, \quad C^{1}$ in $\mathbb{R}^{n} \backslash \stackrel{\circ}{\mathcal{T}}$, positive definite, proper on $\mathcal{T}^{c}$, such that $\forall x \in \mathcal{T}^{c}$,

$$
\max _{\left(w_{0}, w\right) \in S(A)}\left\{\left\langle\nabla U(x), \bar{f}\left(x, w_{0}, w\right)\right\rangle\right\} \leq-m(\mathbf{d}(x))
$$

for some continuous, increasing function $m:] 0,+\infty[\rightarrow] 0,+\infty[$.

A second explicit condition that implies (4.11), involving just the cost, is

(SC2) There are some continuous, increasing functions $\left.c_{1}, c_{2}:\right] 0,+\infty[\rightarrow] 0,+\infty\left[\right.$ and some constant $C_{1} \geq 0$ such that

$$
l(x, a) \geq \max \left\{c_{1}(\mathbf{d}(x)), c_{2}(\mathbf{d}(x))|a|^{q}-C_{1}\right\} \quad \forall(x, a) \in \mathcal{T}^{c} \times A .
$$

In (SC2) we can replace (4.13) by the following stronger hypothesis:

$$
l(x, a) \geq c_{1}(\mathbf{d}(x))+c_{2}(\mathbf{d}(x))|a|^{q} \quad \forall(x, a) \in \mathcal{T}^{c} \times A .
$$

In fact, both (4.13), (4.14) imply that $\forall\left(w_{0}, w\right) \in \Gamma \backslash \Gamma(x)$, one has $\inf _{s \geq 0} c_{i}\left(\mathbf{d}\left(\xi_{x}\left(s, w_{0}, w\right)\right)\right) \geq \bar{c}$ for some $\bar{c}>0$, for $i=1,2$. Hence $J\left(+\infty, x, w_{0}, w\right)=+\infty$ in correspondence of such controls, so that (4.11) turns out to be satisfied.

Notice that, in the special case $c_{2}(\mathbf{d}(x)) \equiv c_{2}>0$ and $q>p,(4.13)$ is assumed in many papers devoted to the uniqueness issue and includes the infinite horizon LQR problem (seen as an asymptotic exit-time problem with $\mathcal{T}=\{0\}$ ), as e.g. [8]. A previous paper of ours, [15], treats the exit-time problem for a weakly coercive Lagrangian, where $(4.14)$ holds for $p=q=1$ and $c_{1}(\cdot), c_{2}(\cdot)$ are bounded below by a positive constant.

Remark 4.8. If we only have the target-weighted coercivity condition (1.4), then for all controls belonging to $\Gamma \backslash \Gamma(x)$, one has $J\left(s, x, w_{0}, w\right) \geq \bar{c} \int_{0}^{s}|w(s)|^{q} \mathrm{~d} s-C_{1} \int_{0}^{s} w_{0}^{q}(s) \mathrm{d} s$ for any $s \geq 0$. Therefore controls such that $\int_{0}^{+\infty} w_{0}^{q}(s) \mathrm{d} s<+\infty$ (implying $\left.\int_{0}^{+\infty}|w(s)|^{q} \mathrm{~d} s=+\infty\right)$, have an infinite cost. As a consequence, (4.11) is satisfied as soon as it holds just for controls in $\Gamma \backslash \Gamma(x)$ such that $\int_{0}^{+\infty} w_{0}^{q}(s) \mathrm{d} s=+\infty$. Hypothesis $l(x, a) \geq c_{1}(\mathbf{d}(x))$ is merely a simple, explicit sufficient condition to this aim.

\subsubsection{Conditions for $V \equiv V^{m, r}$}

For any $x \in \mathcal{T}^{c}$, let us define the relaxed version of $V$,

$$
V^{r}(x) \doteq \inf _{\mu \in \Gamma^{r}(x)} \int_{0}^{\sigma_{x}^{r}(\mu)} \bar{l}^{r}\left(\xi_{x}^{r}(s, \mu), \mu\right) \mathrm{d} s \quad(\leq+\infty),
$$

where $\Gamma^{r}(x) \doteq\left\{\mu \in \Gamma^{r}: \quad \liminf _{s \rightarrow \sigma^{r} \frac{-}{x}(\mu)} \mathbf{d}\left(\xi_{x}^{r}(s, \mu)\right)=0\right\}$. In order to have $V=V^{m, r}$, it is clearly sufficient to give conditions implying either that $V \equiv V^{m}$ and $V^{m} \equiv V^{m, r}$ or that $V^{r} \equiv V^{m, r}$ and $V \equiv V^{r}$. Since for any $x \in \mathbb{R}^{n}$,

$$
\overline{c o}\left(\bar{f}(x, S(A)) \times \bar{l}(x, S(A))=\bar{f}^{r}\left(x, A^{r}\right) \times \bar{l}^{r}\left(x, A^{r}\right),\right.
$$

standard arguments yield that, if the set

$$
\begin{aligned}
L(x) \doteq\{ & (\lambda, \gamma) \in \mathbb{R}^{n+1}: \exists w \in \overline{B(0,1)} \cap A \text { s.t. } \\
& \left.\left.\quad \lambda=\bar{f}\left(x,\left(1-|w|^{q}\right)^{1 / q}, w\right), \quad \bar{l}\left(x,\left(1-|w|^{q}\right)^{1 / q}, w\right)\right) \leq \gamma\right\}
\end{aligned}
$$

is convex for any $x \in \mathcal{T}^{c}$, then $V^{r} \equiv V$ and $V^{m, r} \equiv V^{m}$. Actually, in several situations these equalities are true under convexity assumptions weaker than (4.16), that are satisfied, for instance, when $f$ is affine and $l$ is convex in the control variable. Precisely, as established in the next propositions, $V^{r} \equiv V$ under the following condition, where the (non convex) control set $S(A)$ is replaced by $[0,1] \times \overline{B(0,1)} \cap A$. 
(CV) For any $x \in \mathcal{T}^{c}$, the set:

$$
\begin{gathered}
L^{\prime}(x) \doteq\left\{(\lambda, \gamma) \in \mathbb{R}^{n+1}: \exists\left(w_{0}, w\right) \in[0,1] \times(\overline{B(0,1)} \cap A),\right. \text { s.t. } \\
\left.\left.\lambda=\bar{f}\left(x, w_{0}, w\right) \quad \bar{l}\left(x, w_{0}, w\right)\right) \leq \gamma\right\}
\end{gathered}
$$

is convex.

Proposition 4.9. Assume (CV). Then $V^{r} \equiv V$.

Proof. Let $x \in \mathcal{T}^{c}$ be such that $V^{r}(x)<+\infty$. In order to prove that $V(x)=V^{r}(x)$, let $\mu \in \Gamma^{r}(x)$ be an arbitrary relaxed control such that

$$
J^{r}\left(\sigma_{x}^{r}(\mu), x, \mu\right) \doteq \int_{0}^{\sigma_{x}^{r}(\mu)} \bar{l}^{r}\left(\xi_{x}^{r}(s, \mu), \mu\right) \mathrm{d} s<+\infty .
$$

Thanks to $(\mathrm{CV})$, by standard arguments there exists a control $\left(w_{0}, w\right) \in \mathcal{B}\left(\mathbb{R}_{+},[0,1] \times(\overline{B(0,1)} \cap A)\right)$ such that $\xi_{x}^{r}(\cdot, \mu) \equiv \xi_{x}\left(\cdot, w_{0}, w\right), \sigma_{x}^{r}(\mu)=\sigma_{x}\left(w_{0}, w\right)$ and $J^{r}\left(\sigma_{x}^{r}(\mu), x, \mu\right) \geq J\left(\sigma_{x}\left(w_{0}, w\right), x, w_{0}, w\right)$. We point out that $\left(w_{0}, w\right) \notin \Gamma(x)$ in general, since $w_{0}^{q}+|w|^{q}$ might be strictly smaller than 1 . Nevertheless, as soon as

$$
\int_{0}^{\sigma_{x}\left(w_{0}, w\right)}\left[w_{0}^{q}(s)+|w(s)|^{q}\right] \mathrm{d} s>0,
$$

using the arc-lenght reparameterization $\Phi^{-1}$, where $\Phi(\sigma)=\int_{0}^{\sigma}\left[w_{0}^{q}(s)+|w(s)|^{q}\right] \mathrm{d} s$, the control $\left(w_{0}, w\right)$ can be substituted by one taking values in $S(A)$ having the same cost and trajectory, since $\bar{f}$ and $\bar{l}$ are $q$-positively homogeneous in $\left(w_{0}, w\right)$. Condition (4.18) is surely satisfied, since, if $\left(w_{0}, w\right)=0$ a.e., we would have $\xi_{x}\left(s, w_{0}, w\right)=x \notin \mathcal{T}$ for all $s \geq 0$, in contradiction with the fact that $\liminf _{s \rightarrow \sigma_{x}^{-}\left(w_{0}, w\right)} \mathbf{d}\left(\xi_{x}\left(s, w_{0}, w\right)\right)=0$.

The equality $V^{m, r} \equiv V^{m}$ holds when, for instance, either (1.3) or (1.4) for $q \geq p$ is in force and we have the following condition, stronger than (CV) since we consider now the space-time dynamics $\left(w_{0}, \bar{f}\left(x, w_{0}, w\right)\right)$.

$(\mathbf{C V})^{\prime}$ For any $x \in \mathcal{T}^{c}$, the set:

$$
\begin{gathered}
L^{\prime}(x) \doteq\left\{\left(\lambda_{0}, \lambda, \gamma\right) \in \mathbb{R}^{1+n+1}: \exists\left(w_{0}, w\right) \in[0,1] \times(\overline{B(0,1)} \cap A), \quad\right. \text { s.t. } \\
\left.\left.\left(\lambda_{0}, \lambda\right)=\left(w_{0}, \bar{f}\left(x, w_{0}, w\right)\right) \quad \bar{l}\left(x, w_{0}, w\right)\right) \leq \gamma\right\}
\end{gathered}
$$

is convex.

Proposition 4.10. Assume $(\mathrm{CV})^{\prime}$ and either (1.3) or (1.4) for $q \geq p$. Then $V^{m, r} \equiv V^{m}$.

Proof. Arguing as in the previous proposition, for any $x \in \mathcal{T}^{c}$ and $\mu \in \Gamma^{r}$ such that $J^{r}\left(\sigma_{x}^{r}(\mu), x, \mu\right)<+\infty$, in view of $(\mathrm{CV})^{\prime}$ there is a control $\left(w_{0}, w\right) \in \mathcal{B}\left(\mathbb{R}_{+},[0,1] \times(\overline{B(0,1)} \cap A)\right)$ such that $\xi(\cdot) \doteq \xi_{x}^{r}(\cdot, \mu) \equiv \xi_{x}\left(\cdot, w_{0}, w\right)$, $\sigma_{x}^{r}(\mu)=\sigma_{x}\left(w_{0}, w\right), J^{r}\left(\sigma_{x}^{r}(\mu), x, \mu\right) \geq J\left(\sigma_{x}\left(w_{0}, w\right), x, w_{0}, w\right)$ and in addition

$$
\int_{0}^{\sigma} w_{0}^{q}(s) \mathrm{d} s=\int_{0}^{\sigma}\left(1-|\mu(s)|^{q}\right) \mathrm{d} s \quad \forall \sigma \geq 0 .
$$

If $\mu \in \Gamma^{r}(x)$, we can conclude as in the proof of Proposition 4.9. If $\mu \notin \Gamma^{r}(x), \sigma_{x}^{r}(\mu)=+\infty$ and applying the arc-lenght reparameterization $\Phi^{-1}$ and the same arguments as above, we obtain a reparametrized control belonging to $\Gamma$ only if

$$
\int_{0}^{+\infty}\left[w_{0}^{q}(s)+|w(s)|^{q}\right] \mathrm{d} s=+\infty
$$


In fact by (4.20) if we had $\int_{0}^{+\infty} w_{0}^{q}(s) \mathrm{d} s=T<+\infty$, then it would be $\int_{0}^{+\infty}|\mu(s)|^{q} \mathrm{~d} s=+\infty$. Since $\mu \in$ $\Gamma^{r} \backslash \Gamma^{r}(x)$, then $\left.\inf _{s \geq 0} \mathbf{d}(\xi(s))\right)=\bar{c}>0$ and (1.3) or (1.4) for $q \geq p$ would yield a cost

$$
J^{r}(+\infty, x, \mu) \geq \bar{c} \int_{0}^{+\infty}|\mu(s)|^{q}-C_{1} T=+\infty,
$$

which is a contradiction. Therefore $\int_{0}^{+\infty} w_{0}^{q}(s) \mathrm{d} s=+\infty$, which implies (4.21).

Corollary 4.11. If (CV) and either (SC1) or (SC2) holds, then $V=V^{m, r}$.

Proof. In view of (4.15), both (SC1) and (SC2) (in the extended setting, namely, $\bar{l}\left(x, w_{0}, w\right) \geq$ $\max \left\{c_{1}(\mathbf{d}(x)) w_{0}^{q}, c_{2}(\mathbf{d}(x))|w|^{q}-C_{1} w_{0}^{q}\right\}$ for all $\left.\left(x, w_{0}, w\right)\right)$ hold also for the relaxed framework. Therefore by Section 4.3.1, each one of them yields $V^{r} \equiv V^{m, r}$. The proof is thus concluded, since (CV) implies $V^{r} \equiv V$ by Proposition 4.9 .

\subsubsection{The degenerate case: $l(x, a) \equiv l(x)$}

In this case we have $V^{m, r} \equiv V^{m}$, being $V^{m} \equiv 0$. Nevertheless, as shown in Example 3.11, it might be that $\mathcal{V}$ and $V$ are not zero. Therefore it is interesting to single out some cases in which $V$ and $\mathcal{V}$ are themselves identically zero. It is rather intuitive that this happens if the following hypothesis holds:

$(\mathbf{S C 3}) \mathcal{V}$ is continuous on $\partial \mathcal{T}$ and the control system $\xi^{\prime}(s)=\bar{f}(s, 0, w(s))$, where $w(s) \in A \cap\{w:|w|=1\}$ is asymptotically controllable to $\mathcal{T}$.

In fact, owing to Theorem 3.6, by the continuity of $\mathcal{V}$ on $\partial \mathcal{T}$ we have $\mathcal{V} \equiv V$. Moreover, the asymptotic controllability hypothesis implies the existence of a control $(0, w)$, whose corresponding trajectory approaches asymptotically the target (with zero cost). Therefore $\mathcal{V} \equiv V \equiv 0$.

Example 4.12. By Theorem 2.4 it is easy to see that for an affine dynamics $f(x, a)=f_{0}(x)+\sum_{i=1}^{m} f_{i}(x) a_{i}$, a sufficient condition for (SC3) is the following.

(SC3)' There is some $U: \mathbb{R}^{n} \backslash \stackrel{\circ}{\mathcal{T}} \rightarrow \mathbb{R}_{+}$continuous, locally semiconcave, positive definite and proper on $\mathcal{T}^{c}$, such that, for all $x \in \mathcal{T}^{c}$, one has

$$
\min _{w \in A,|w|=1}\left\langle p, \sum_{i=1}^{m} f_{i}(x) w_{i}\right\rangle<0 \quad \forall p \in D^{*} U(x)
$$

while for all $x \in \mathcal{T}_{\delta}$ for some $\delta>0$ there is some $k>0$ such that:

$$
\left\{\left\langle p, f_{0}(x)\right\rangle+k l(x)\right\} \leq 0 \quad \forall p \in D^{*} U(x) .
$$

\section{Approximations AND STABILITY}

In this section we introduce some value functions $\mathcal{U}^{\rho}$ obtained by minimizing over various subsets $\mathcal{A}_{\rho}(x)$ of the control set $\mathcal{A}(x)$ where, for instance, either $t_{x}(\alpha)<+\infty$ or energy blow-ups are forbidden. The functions $\mathcal{U}^{\rho}$, greater in general than $\mathcal{V}$, turn out to be the limits, as $\varepsilon \rightarrow 0$, of some values $\mathcal{V}_{\varepsilon}^{\rho}$, obtained by minimizing, over $\mathcal{A}(x)$, some penalized lagrangians $l+\varepsilon \rho$. As shown in Theorem 5.7 below, each $\mathcal{U}^{\rho}$ coincides with $\mathcal{V}$ as soon as it is continuous on $\partial \mathcal{T}$. As a consequence, under suitable local asymptotic controllability assumptions, the value function $\mathcal{V}$ can be approximated by $\mathcal{V}_{\varepsilon}^{\rho}$, which, in general, are more regular than $\mathcal{V}$, since the penalized problems can be chosen to be coercive. Moreover, they are the unique nonnegative solutions of the corresponding boundary value problems, $(\mathrm{BVP})_{\varepsilon}$. Such an approximation can be seen as a stability result for the original Hamilton-Jacobi equation for which the comparison property does not hold. 
Precisely, for any $\rho: \mathbb{R}^{n} \times A \rightarrow \mathbb{R}_{+}$continuous and any $\left.\left.\varepsilon \in\right] 0,1\right]$, let us set

$$
\begin{aligned}
& K_{\varepsilon}(x, u, p) \doteq \\
& \max _{\left(w_{0}, w\right) \in S(A)}\left\{-\left\langle p, \bar{f}\left(x, w_{0}, w\right)\right\rangle-\overline{(l+\varepsilon \rho)}\left(x, w_{0}, w\right)+\overline{(l+\varepsilon \rho)}\left(x, w_{0}, w\right) u\right\}
\end{aligned}
$$

(where we tacitly assume that $\Phi \doteq l+\varepsilon \rho$ satisfies (H1)). In view of Theorems 2.4, 4.6 and Corollary 4.11, Theorem 5.7 below implies the following stability result.

Theorem 5.1. Let $\rho: \mathbb{R}^{n} \times A \rightarrow \mathbb{R}_{+}$be a continuous function. Assume (CV) and either (SC1) or (SC2) for $l$ replaced by $l+\rho$ and suppose that there is a local MRF $U$ for $(l+\rho, A)$. Then for any $\varepsilon \in] 0,1]$ there exists a unique nonnegative solution $\mathcal{W}_{\varepsilon}$ to

$$
\begin{cases}K_{\varepsilon}(x, W(x), D W(x))=0 & \text { in } \mathbb{R}^{n} \backslash \mathcal{T} \\ W(x)=0 & \text { on } \partial \mathcal{T} .\end{cases}
$$

Moreover, as $\varepsilon \rightarrow 0^{+}$the $\mathcal{W}_{\varepsilon}$ converge locally uniformly to a function $\mathcal{W}$ such that $\mathcal{V} \equiv-\log (1-\mathcal{W})$ and $\operatorname{Dom}(\mathcal{V})=\{x: \mathcal{W}(x)<1\}$.

Choosing $\rho(x, a) \doteq\left(1+|a|^{r}\right)$ for some integer $r \geq q$ and $r>p$, then $l(x, a)+\rho(x, a)$ satisfies (SC2) for $q$ replaced by $r$ and the extended setting coincides with the original one, since we have coercivity. Of course, Theorem 5.1 applies even to more general $\rho$, as shown in the following example.

Example 5.2. Let us consider again the degenerate cheap control problem of Example 3.11, where $\mathcal{T}=\{0\}$, $f(x, a)=-x+a, l(x, a)=|x|, x \in \mathbb{R}$ and $a \in \mathbb{R}_{+}$. Let us set $\rho(x, a) \doteq|a|$ and consider $\forall \varepsilon>0$ the penalized value function

$$
\mathcal{V}_{\varepsilon}(x)=\inf _{\alpha \in \mathcal{A}(x)} \int_{0}^{t_{x}(\alpha)}|y(t)|+\varepsilon|\alpha(t)| \mathrm{d} t
$$

One easily sees that

$$
\mathcal{V}_{\varepsilon}(x)= \begin{cases}x & \text { if } x \geq 0 \\ \varepsilon|x| & \text { if } x<0\end{cases}
$$

so that the limit as $\varepsilon \rightarrow 0^{+}$coincides with $\mathcal{V}$. Since the convexity hypothesis (CV) and (SC2) hold and all the above value functions are continuous, in view of Theorem 5.1, for every $\varepsilon>0$ the function $\mathcal{V}_{\varepsilon}$ is, the unique nonnegative solution of the associated boundary value problem, say $(\mathrm{BVP})_{\varepsilon}$ (defined as (BVP) with $l$ replaced by $l+\varepsilon \rho$ and $\rho \equiv|a|)$. Moreover, $\mathcal{V}$ is the locally uniform limit of the sequence $\mathcal{V}_{\varepsilon}$ as $\varepsilon \rightarrow 0^{+}$.

Let us now define for every $x \in \mathcal{T}^{c}$ the subset of $\rho$-admissible controls

$$
\mathcal{A}_{\rho}(x) \doteq\left\{\alpha \in \mathcal{A}(x): \quad \int_{0}^{t_{x}(\alpha)} \rho\left(y_{x}(t, \alpha), \alpha(t)\right) \mathrm{d} t<+\infty\right\}
$$

and the corresponding value function

$$
\mathcal{U}^{\rho}(x) \doteq \inf _{\alpha \in \mathcal{A}_{\rho}(x)} \mathcal{J}\left(t_{x}(\alpha), x, \alpha\right)
$$

Example 5.3. Usual choices of the function $\rho$ are

$$
\rho_{1} \equiv 1, \quad \rho_{2} \equiv|a|^{r}, \quad \text { and } \quad \rho_{3} \equiv\left(1+|a|^{r}\right) \quad \text { for some integer } r \geq q \text {, }
$$


whose corresponding limit functions $\mathcal{U}^{\rho_{i}}$ for $i=1,2,3$ are the following value functions $\mathcal{V}^{f}$, $\mathcal{V}^{L^{r}}$, and $\mathcal{V}^{f, L^{r}}$, respectively:

$$
\begin{aligned}
& \mathcal{V}^{f}(x) \doteq \inf _{\left\{\alpha \in \mathcal{A}(x): t_{x}(\alpha)<+\infty\right\}} \mathcal{J}\left(t_{x}(\alpha), x, \alpha\right) \quad \forall x \in \mathcal{T}^{c}, \\
& \mathcal{V}^{L^{r}}(x) \doteq \inf _{\alpha \in L^{r}\left(\mathbb{R}_{+}, A\right) \cap \mathcal{A}(x)} \mathcal{J}\left(t_{x}(\alpha), x, \alpha\right) \quad \forall x \in \mathcal{T}^{c}, \\
& \mathcal{V}^{f, L^{r}}(x) \doteq \inf _{\left\{\alpha \in L^{r}\left(\mathbb{R}_{+}, A\right) \cap \mathcal{A}(x): t_{x}(\alpha)<+\infty\right\}} \mathcal{J}\left(t_{x}(\alpha), x, \alpha\right) \quad \forall x \in \mathcal{T}^{c} .
\end{aligned}
$$

The finite-time-and-energy exit-time value function $\mathcal{V}^{f, L^{r}}$, in particular, plays an important role for the approximation issue, since it is the natural limit of penalized problems associated to HJB equations satisfying a comparison principle. For instance, in Example 5.2 above, $\mathcal{V}$ turns out to be approximated by the functions $\mathcal{V}_{\varepsilon}$ since it coincides with $\mathcal{V}^{L^{1}}$.

In general, $\mathcal{V} \leq \mathcal{U}^{\rho}$ and the inequality may be strict. In Theorem 5.5 below we show that $\mathcal{V} \equiv \mathcal{U}^{\rho}$, as soon as $\mathcal{U}^{\rho}$ is continuous on $\partial \mathcal{T}$.

In the sequel we need the following density result, crucial in order to consider functions $\rho$ with arbitrary growth in the control variable.

Proposition 5.4. Let $T>0$. For any control $\alpha \in L^{q}([0, T], A)$ there exists a sequence of controls $\alpha_{N} \in$ $L^{\infty}([0, T], A \cap \overline{B(0, N)})$ for all $N \in \mathbb{N}$, verifying:

$$
\begin{aligned}
& \lim _{N \rightarrow+\infty}\left|\alpha_{N}(t)\right|=|\alpha(t)|, \text { and }\left|\alpha_{N}(t)\right| \leq|\alpha(t)| \text { for a.e. } t \in[0, T], \\
& \lim _{N \rightarrow+\infty} \sup _{t \in[0, T]}\left|y_{x}\left(t, \alpha_{N}\right)-y_{x}(t, \alpha)\right|=0, \\
& \lim _{N \rightarrow+\infty} \mathcal{J}\left(T, x, \alpha_{N}\right)=\mathcal{J}(T, x, \alpha) .
\end{aligned}
$$

The proof of Proposition 5.4 is postponed to the Appendix.

Theorem 5.5. If $\mathcal{U}^{\rho}$ is continuous on $\partial \mathcal{T}$, then $\mathcal{U}^{\rho} \equiv \mathcal{V}$.

Proof. Let $x \in \mathcal{T}^{c}$. The inequality $\mathcal{V}(x) \leq \mathcal{U}^{\rho}(x)$ is obvious. Hence, if $\mathcal{V}(x)=+\infty, \mathcal{U}^{\rho}(x)=+\infty$ too. Let $\mathcal{V}(x)<+\infty$. Fix $\eta>0$. By the continuity of $\mathcal{U}^{\rho}$ on the compact set $\partial \mathcal{T}$, there is some $\delta>0$ such that

$$
\mathcal{U}^{\rho}(\bar{x})<\eta \quad \forall \bar{x} \in \mathcal{T}^{c} \quad \text { with } \quad \mathbf{d}(\bar{x})<\delta .
$$

By the definition of $\mathcal{V}$, there exists a control $\tilde{\alpha} \in \mathcal{A}(x)$ such that

$$
\int_{0}^{t_{x}(\tilde{\alpha})} l\left(y_{x}(t, \tilde{\alpha}), \tilde{\alpha}(t)\right) \mathrm{d} t<\mathcal{V}(x)+\eta, \quad \mathbf{d}\left(y_{x}(\bar{t}, \tilde{\alpha})\right)<\delta / 2
$$

for some $\bar{t}<t_{x}(\tilde{\alpha})$. Since $\tilde{\alpha} \in L^{q}([0, \bar{t}], A)$, in view of Proposition 5.4 we can approximate on $[0, \bar{t}]$ the control $\tilde{\alpha}$ by a compact-valued control $\hat{\alpha}$ such that $\int_{0}^{\bar{t}}|\hat{\alpha}(t)|^{q} \mathrm{~d} t \leq \int_{0}^{\bar{t}}|\tilde{\alpha}(t)|^{q} \mathrm{~d} t$ and

$$
\int_{0}^{\bar{t}} l\left(y_{x}(t, \hat{\alpha}), \hat{\alpha}(t)\right) \mathrm{d} t<\mathcal{V}(x)+2 \eta, \quad \mathbf{d}\left(y_{x}(\bar{t}, \hat{\alpha})\right)<\delta .
$$

Now by estimate (2.5), there is some $R>0$ (depending just on $\bar{t}$ and $\int_{0}^{\bar{t}}|\tilde{\alpha}(t)|^{p} \mathrm{~d} t$ ) such that $\left|y_{x}(t, \hat{\alpha})\right| \leq R$ for all $t \in[0, \bar{t}]$. Hence $\int_{0}^{\bar{t}} \rho\left(y_{x}(t, \hat{\alpha}), \hat{\alpha}(t)\right) \mathrm{d} t<+\infty$ because of the continuity of $\rho$ together with the boundedness of $\hat{\alpha}$. Let $\bar{x} \doteq y_{x}(\bar{t}, \hat{\alpha})$ and let $\bar{\alpha} \in \mathcal{A}_{\rho}(\bar{x})$ be a control such that $\int_{0}^{t_{\bar{x}}(\bar{\alpha})} l\left(y_{\tilde{x}}(t, \bar{\alpha}), \bar{\alpha}(t)\right) \mathrm{d} t<\eta$, which exists in view of (5.3). Then, the control $\alpha(t)=\hat{\alpha}(t) \chi_{[0, \bar{t}]}(t)+\bar{\alpha}(t-\bar{t}) \chi_{] \bar{t},+\infty]}(t)$ belongs to $\mathcal{A}_{\rho}(x)$ and

$$
\int_{0}^{t_{x}(\alpha)} l\left(y_{x}(t, \alpha), \alpha(t)\right) \mathrm{d} t<\mathcal{V}(x)+3 \eta,
$$

so that $\mathcal{U}^{\rho}(x)<\mathcal{V}(x)+3 \eta$. The proof of the theorem is thus concluded, by the arbitrariness of $\eta>0$. 
Remark 5.6. Another set of controls containing $\mathcal{A}_{\rho}(x)$, interesting for our minimization problems, is given by the following

$$
\mathcal{A}_{\rho}^{\mathrm{loc}}(x) \doteq\left\{\alpha \in \mathcal{A}(x): \quad \int_{0}^{t_{x}(\alpha)} \rho\left(y_{x}(t, \alpha), \alpha(t)\right) \mathrm{d} t<+\infty \quad \text { if } t_{x}(\alpha)<+\infty\right\} .
$$

Indeed, for instance if $\rho \equiv|a|^{q}$, then $\mathcal{A}_{\rho}^{\text {loc }}(x)=L_{\text {loc }}^{q}\left(\mathbb{R}_{+}, A\right) \cap \mathcal{A}(x)$ for any $x \in \mathcal{T}^{c}$. If we denote by $\tilde{\mathcal{A}}(x)$ either $\mathcal{A}_{\rho}(x)$ or $\mathcal{A}_{\rho}^{\text {loc }}(x)$ and by $\mathcal{U}^{\tilde{\mathcal{A}}}(x) \doteq \inf _{\alpha \in \tilde{\mathcal{A}}(x)} \mathcal{J}\left(t_{x}(\alpha), x, \alpha\right)$ the corresponding value function, then, by the proof of Theorem 5.5, it is easy to see the $\mathcal{V} \equiv \mathcal{U}^{\tilde{\mathcal{A}}}$ when $\mathcal{U}^{\tilde{\mathcal{A}}}$ is continuous on $\partial \mathcal{T}$.

Let now $\varepsilon>0$. For every $x \in \mathcal{T}^{c}$ and $\alpha \in \mathcal{A}(x)$, we define the $\varepsilon$-penalized payoff,

$$
\mathcal{J}_{\varepsilon}^{\rho}(t, x, \alpha) \doteq \int_{0}^{t}\left[l\left(y_{x}(\tau, \alpha), \alpha(\tau)\right)+\varepsilon \rho\left(y_{x}(\tau, \alpha), \alpha(\tau)\right)\right] \mathrm{d} \tau
$$

and the corresponding $\varepsilon$-penalized value function,

$$
\mathcal{V}_{\varepsilon}^{\rho}(x) \doteq \inf _{\alpha \in \mathcal{A}(x)} \mathcal{J}_{\varepsilon}\left(t_{x}(\alpha), x, \alpha\right)
$$

As in the case of bounded controls, penalized problems converge in general to $\mathcal{U}^{\rho} \geq \mathcal{V}$. Owing to Theorem 5.5, the limit does coincide with $\mathcal{V}$ when $\mathcal{U}^{\rho}$ is continuous on $\partial \mathcal{T}$.

Theorem 5.7. Let $\rho: \mathbb{R}^{n} \times A \rightarrow \mathbb{R}_{+}$be a continuous function. Then

(i) we have

$$
\lim _{\varepsilon \rightarrow 0^{+}} \mathcal{V}_{\varepsilon}^{\rho}(x)=\mathcal{U}^{\rho}(x) \quad \forall x \in \mathcal{T}^{c}
$$

(ii) If there is some $\bar{\varepsilon}>0$ such that $\mathcal{V}_{\bar{\varepsilon}}^{\rho}$ is continuous on $\partial \mathcal{T}$ and $\mathcal{V}$ is continuous on its whole domain, then

$$
\lim _{\varepsilon \rightarrow 0^{+}} \mathcal{V}_{\varepsilon}^{\rho}=\mathcal{V} \quad \text { uniformly on any compact set } Q \subset \operatorname{Dom}(\mathcal{V})
$$

We omit the proof, since it is the same as in the case of bounded controls (see Thm. 3.3 in [16]).

A sufficient condition for the continuity of $\mathcal{U}^{\rho}$ on $\partial \mathcal{T}$, based on the existence of a special MRF for $\left(l, A^{\prime}\right)$ for some compact subset $A^{\prime} \subset A$, can be found in [16], Theorem 4.2.

The global continuity of $\mathcal{V}$ holds under the assumptions of either Theorem 6.1 or 4.6 , while, owing to Theorem 2.4 below and Remark 2.5, a condition for the continuity of the $\mathcal{V}_{\varepsilon}^{\rho}$ on $\partial \mathcal{T}$ (which yields also the continuity of $\mathcal{U}^{\rho}$ on $\left.\partial \mathcal{T}\right)$ is the existence of a local MRF $U$ for $(l+\rho, A)$.

Incidentally, Theorem 2.4 also implies that if we have a local MRF $U$ for $(l, A)$, then the $\mathcal{V}_{\varepsilon}^{\rho}$ corresponding to $\rho(x, a) \doteq m(U(x)) / k$ are continuous on $\partial \mathcal{T}$ for all $\varepsilon \in] 0,1]$ (for $m$ and $k$ defined as in Thm. 2.4).

\section{Continuity}

In this section we introduce sufficient conditions in order to propagate the continuity of $\mathcal{V}$ from the target to its whole domain. Since we do not assume any convexity hypothesis, such a result cannot be deduced from Theorem 4.6.

Hypothesis (TPK) below, loosely speaking says that for any fixed $\delta$ - neighborhood of the target, for every $x \in \operatorname{Dom}(\mathcal{V})$ one can select a nearly optimal asymptotic trajectory reaching $\mathcal{T}_{\delta}$ in finite time $T$ and with finite $L^{q}$-fuel $K$, depending just on $\mathcal{V}(x)$ and $\delta$ (that is, uniformly w.r.t. $x$ and $\alpha$ ). It can be seen as a kind of turnpike condition with respect to the target $\mathcal{T}$, where the quite unusual fuel constraint takes into account the unboundedness of the control system (see $[5,16]$ ). 
(TPK) $\forall R, \eta, \delta>0$, there exist some increasing functions $T(\cdot), K(\cdot): \mathbb{R}_{+} \rightarrow \mathbb{R}_{+}$such that for every $x \in \mathcal{T}_{\delta}^{c} \cap \operatorname{Dom}(\mathcal{V}), \mathbf{d}(x) \leq R$, there is a control $\alpha \in \mathcal{A}(x)$ verifying

$$
\int_{0}^{t_{x}(\alpha)} l\left(y_{x}(t, \alpha), \alpha(t)\right) \mathrm{d} t \leq \mathcal{V}(x)+\eta
$$

and

$$
\begin{aligned}
& t_{x}^{\delta}(\alpha) \doteq \inf \left\{t>0: y_{x}(t, \alpha) \in \mathcal{T}_{\delta}\right\} \leq T(\mathcal{V}(x)), \\
& \int_{0}^{t_{x}^{\delta}(\alpha)}|\alpha(t)|^{q} \mathrm{~d} t \leq K(\mathcal{V}(x)) .
\end{aligned}
$$

Theorem 6.1. Assume (TPK). If $\mathcal{V}$ is continuous on $\partial \mathcal{T}$, then it is continuous in its domain and it satisfies (4.4).

The above result can be stated using the explicit conditions introduced in Subsection 4.3.1.

Proposition 6.2. Assume $\mathcal{V}$ continuous on $\partial \mathcal{T}$. Then either $(\mathrm{SC} 1)$ or $(\mathrm{SC} 2)$ yields that $\mathcal{V}$ is continuous on $\operatorname{Dom}(\mathcal{V})$ and it satisfies (4.4).

We postpone the proof of the above proposition to the end of the section.

Proof of Theorem 6.1.

In view of Proposition 2.6, $\operatorname{Dom}(\mathcal{V})$ is an open set of $\mathcal{T}^{c}$ where $\mathcal{V}$ is locally bounded and upper semicontinuous. Let $x_{0} \in \operatorname{Dom}(\mathcal{V})$ and let $\nu>0$ be such that $B\left(x_{0}, \nu\right) \subset \operatorname{Dom}(\mathcal{V})$. Let $M \doteq \sup \left\{\mathcal{V}(x): x \in B\left(x_{0}, \nu\right)\right\}+3$, $R \doteq \mathbf{d}\left(x_{0}\right)+\nu$. Fix $\left.\eta \in\right] 0,1\left[\right.$. By the continuity of $\mathcal{V}$ on the compact set $\partial \mathcal{T}$, there is some $\delta \equiv \delta_{\eta}>0$ such that for any $x \in \mathcal{T}_{2 \delta}$ there is a control $\tilde{\alpha} \in \mathcal{A}(x)$ verifying

$$
\int_{0}^{t_{x}(\tilde{\alpha})} l\left(y_{x}(t, \tilde{\alpha}), \tilde{\alpha}(t)\right) \mathrm{d} t \leq \eta .
$$

Fix $x_{1}, x_{2} \in B\left(x_{0}, \nu\right)$ and assume e.g. $\mathcal{V}\left(x_{2}\right) \geq \mathcal{V}\left(x_{1}\right)$. By (TPK) there exist some $T=T(M), K=K(M)$ and an $\eta$-optimal control for $\mathcal{V}\left(x_{1}\right), \alpha_{1} \in \mathcal{A}\left(x_{1}\right)$, so that

$$
\int_{0}^{t_{x_{1}}\left(\alpha_{1}\right)} l\left(y_{x_{1}}\left(t, \alpha_{1}\right), \alpha_{1}(t)\right) \mathrm{d} t \leq \mathcal{V}\left(x_{1}\right)+\eta<M,
$$

and, for some $\bar{t} \leq T \wedge t_{x_{1}}\left(\alpha_{1}\right)$, we get

$$
\mathbf{d}\left(y_{x_{1}}\left(\bar{t}, \alpha_{1}\right)\right)<\delta \text { and } \int_{0}^{\bar{t}}\left|\alpha_{1}(t)\right|^{q} \mathrm{~d} t \leq K
$$

Recalling that $q \geq p$, there is some positive constant, denoted again by $K$, such that the second inequality above holds also for $p$. By (2.5) and (2.6) all trajectories starting from points $x \in B\left(x_{0}, \nu\right)$ and corresponding to a control $\alpha$ such that $\int_{0}^{T}|\alpha(t)|^{p} d t \leq K$, verify $\left|y_{x}(t, \alpha)\right| \leq R \quad \forall t \in[0, T]$ for some $R>0$. Moreover,

$$
\left|y_{x_{2}}(t, \alpha)-y_{x_{1}}(t, \alpha)\right| \leq L\left|x_{2}-x_{1}\right| \quad \forall t \in[0, T], \quad \forall x_{1}, x_{2} \in B\left(x_{0}, \nu\right),
$$

for a suitable $L>0$. Therefore, choosing $0<\nu^{\prime}<\nu$ small enough, for $x_{1}, x_{2} \in B\left(x_{0}, \nu^{\prime}\right)$ we get

$$
\begin{aligned}
& 0 \leq \mathcal{V}\left(x_{2}\right)-\mathcal{V}\left(x_{1}\right) \leq \int_{0}^{\bar{t}}\left|l\left(y_{x_{2}}\left(t, \alpha_{1}\right), \alpha_{1}(t)\right)-l\left(y_{x_{1}}\left(t, \alpha_{1}\right), \alpha_{1}(t)\right)\right| d t+3 \eta \\
& \leq T \omega\left(L\left|x_{2}-x_{1}\right|, R\right)+3 \eta<4 \eta,
\end{aligned}
$$

which implies the continuity of $\mathcal{V}$ by the arbitrariness of $\eta>0$. The boundary condition (4.4) can be obtained arguing as in the proof of Theorem 4.1 in [16]. 
The fuel constraint in (6.2) can be dropped in (TPK) if we consider the weak target-weighted coercivity condition (1.4). In this case, (TPK) turns out to be equivalent to the following more usual turnpike condition, $(\mathrm{TPK})^{\prime}$, introduced in [16] in the case of bounded controls.

$(\mathbf{T P K})^{\prime} \forall R, \eta, \delta>0$, there exists some increasing function $T(\cdot): \mathbb{R}_{+} \rightarrow \mathbb{R}_{+}$such that for every $x \in$ $\mathcal{T}_{\delta}^{c} \cap \operatorname{Dom}(\mathcal{V}), \mathbf{d}(x) \leq R$, there is a control $\alpha \in \mathcal{A}(x)$ satisfying (6.1) such that $t_{x}^{\delta}(\alpha) \leq T(\mathcal{V}(x))$.

Indeed, if (1.4) holds, then $\forall x \in \mathcal{T}_{\delta}^{c}$ and $\forall \alpha \in \mathcal{A}$ satisfying (6.1) one has

$$
\int_{0}^{t_{x}^{\delta}(\alpha)}|\alpha(t)|^{q} \mathrm{~d} t \leq K \doteq \frac{\mathcal{V}(x)+\eta+C_{1} t_{x}^{\delta}(\alpha)}{c_{2}(\delta)} .
$$

Let us remark that $(\mathrm{TPK})^{\prime}$ is not sufficient to propagate the continuity of $\mathcal{V}$ from $\partial \mathcal{T}$, when the $L^{q}$-norm of minimizing control sequences diverge to $+\infty$.

Proof of Proposition 6.2. It is easy to see that (1.4) yields (TPK)', therefore (SC2) is a sufficient condition for (TPK) and by Theorem 6.1 the thesis is verified.

Otherwise, when $\mathcal{V}$ is continuous on $\partial \mathcal{T}$, assumption (SC1) implies (TPK) and therefore, the global continuity of $\mathcal{V}$. In fact, fix $R, \eta$ and $\delta>0$. Then (SC1), sufficient for the UGAS property of the extended control system, implies the existence (see e.g. [1]) of a $\mathcal{K} \mathcal{L}$ function $\beta$ such that for every $x$ with $\mathbf{d}(x)<R$, one has $\mathbf{d}\left(\xi_{x}\left(s, w_{0}, w\right)\right) \leq \beta(R, s)$ for all $s \geq 0$ and for any control $\left(w_{0}, w\right) \in \Gamma$. Let $\bar{s}>0$ be the minimum $s$ such that $\beta(R, s)=\delta$. Then, independently from the payoff, one has that $\mathbf{d}\left(\xi_{x}\left(s, w_{0}, w\right)\right)<\delta$ for all $s \geq \bar{s}$. Since this is true in particular for all $\left(w_{0}, w\right) \in \Gamma^{+}$, in view of Proposition 3.1 it implies (6.2) for all $\alpha \in \mathcal{A}$, by choosing $T=K \doteq \bar{s}$.

\section{Appendix. A}

Proof of Proposition 5.4. For every $\alpha \in L^{q}([0, T], A)$, following the construction of Remark 3.1 let us define $s(t) \doteq \int_{0}^{t}\left(1+|\alpha(\tau)|^{q}\right) \mathrm{d} \tau \quad \forall t \in[0, T], S \doteq s(T)$ and consider the extended control $\left(w_{0}, w\right)$ defined by

$$
w(s) \doteq \frac{\alpha(t(s))}{\left(1+|\alpha(t(s))|^{q}\right)^{\frac{1}{q}}}, \quad w_{0}(s) \doteq\left(1-|w(s)|^{q}\right)^{\frac{1}{q}} \quad \forall s \in[0, S],
$$

where $t(s)$ is the inverse function of $s(t)$. For $n \in \mathbb{N}$ let $\left(w_{0_{n}}, w_{n}\right)$ be the control given by

$$
w_{n}(s) \doteq \frac{n}{n+1} w(s), \quad w_{0_{n}}(s) \doteq\left(1-\left|w_{n}(s)\right|^{q}\right)^{\frac{1}{q}} \quad \forall s \in[0, S] .
$$

Let us set $t_{n}(s) \doteq \int_{0}^{s} w_{0_{n}}(\sigma)^{q} \mathrm{~d} \sigma$ for $s \in[0, S], T_{n} \doteq t_{n}(S)(>T)$, and let us denote by $s_{n}(t)$ its inverse $\forall t \in\left[0, T_{n}\right]$. The controls

$$
\alpha_{n}(t) \doteq \frac{w_{n}\left(s_{n}(t)\right)}{w_{0_{n}}\left(s_{n}(t)\right)} \quad \forall t \in[0, T]
$$

form a sequence that satisfies the claim. In fact, $\left|\alpha_{n}\right|^{q}=\frac{\left(\frac{n}{n+1}|w|\right)^{q}}{1-\left(\frac{n}{n+1}|w|\right)^{q}} \leq \frac{n^{q}}{(n+1)^{q}-n^{q}} \leq n^{q}$ and $\left|\alpha_{n}\right|^{q} \leq|\alpha|^{q}$. Let us denote by $y_{n}(\cdot) \doteq y_{x}\left(\cdot, \alpha_{n}\right), y(\cdot) \doteq y_{x}(\cdot, \alpha)$. If $s_{n}$ is such that $t_{n}\left(s_{n}\right)=T$ then $s_{n}=S\left(1-\frac{1}{n}\right)$ and

$$
\begin{array}{r}
\sup _{t \in[0, T]}\left|y_{n}(t)-y(t)\right|=\sup _{s \in[0, S]}\left|y_{n}(t(s))-y(t(s))\right| \\
=\sup _{s \in[0, S]}\left|\tilde{\xi}_{n}(s)-\xi(s)\right|,
\end{array}
$$


where by the correspondence between the solutions to $(2.3)$ and those to $(3.3)$ we have that $y(t(\cdot))=\xi(\cdot) \doteq$ $\xi_{x}\left(\cdot, w_{0}, w\right), y_{n}(t(\cdot))=\tilde{\xi}_{n}(\cdot) \doteq \xi_{n}\left(\left(s_{n} \circ t\right)(\cdot)\right)$, setting $\xi_{n}(\cdot) \doteq \xi_{x}\left(\cdot, w_{0_{n}}, w_{n}\right)$. Since there exists $R>0$ such that

$$
\sup _{0 \leq s \leq S}\left\{\left|\xi_{n}(s)\right|,\left|\tilde{\xi}_{n}(s)\right|,|\xi(s)|\right\} \leq R,
$$

thanks to (2.1) in (H0) and (H1), by the Dominated Convergence Theorem and Gronwall's inequality, standard calculations imply that there exist a sequence $\varepsilon_{n}$ approaching 0 and $L>0$ such that

$$
\sup _{t \in[0, T]}\left|y_{n}(t)-y(t)\right| \leq \varepsilon_{n} \mathrm{e}^{S L} .
$$

As for the cost, observing that $s_{n} \leq S$, by (A.2), (A.3) and (H1), the Dominated Convergence Theorem lets us conclude that

$$
\lim _{n \rightarrow \infty} \mathcal{J}\left(T, x, \alpha_{n}\right)=\mathcal{J}(T, x, \alpha) .
$$

Remark A.1. If (H1) does not hold, Proposition 5.4 can be proven even assuming either that $\rho$ verifies, for any $R>0$,

$$
0 \leq \rho(x, a) \leq \bar{M}_{R}\left(1+|a|^{q}\right) \quad \forall(x, a) \in \mathbb{R}^{n} \times A \text { with }|x| \leq R,
$$

for some $\bar{M}_{R}>0$; or that $f$ and $l$ verify, for any $R>0$,

$$
\begin{aligned}
& \left|f\left(x, a_{1}\right)-f\left(x, a_{2}\right)\right| \leq \bar{M}_{R}\left[1+\left(\left|a_{1}\right| \wedge\left|a_{2}\right|\right)^{p-1}\right]\left|a_{1}-a_{2}\right|, \\
& \left|l\left(x, a_{1}\right)-l\left(x, a_{2}\right)\right| \leq \bar{M}_{R}\left[1+\left(\left|a_{1}\right| \wedge\left|a_{2}\right|\right)^{q-1}\right]\left|a_{1}-a_{2}\right|
\end{aligned}
$$

$\forall a_{1}, a_{2} \in A$ and $x \in \mathbb{R}^{n}$ with $|x| \leq R$, for some $\bar{M}_{R}>0$.

In particular, when (H1) does not hold the density result remains true if we allow penalizations with at most the same growth $q$ of $l$ in the control variable. Incidentally, this restriction does not prevent us to introduce coercive penalizations for some noncoercive problems. Consider, for instance, $f(x, a)$ control-affine, $l(x, a)=$ $|a x|^{2}(1+\sin (x a))$ and the penalization $\rho(x, a)=|a|^{2}$ in $\mathbb{R} \times \mathbb{R}$.

\section{REFERENCES}

[1] A. Bacciotti, Andrea and L. Rosier, Liapunov functions and stability in control theory. Second edition. Commun. Control Engrg. Ser. Springer-Verlag, Berlin (2005).

[2] M. Bardi and I. Capuzzo Dolcetta, Optimal control and viscosity solutions of Hamilton-Jacobi-Bellman equations. Birkhäuser, Boston (1997).

[3] A. Bressan and B. Piccoli, Introduction to the mathematical theory of control. Vol. 2. AIMS Ser. Appl. Math. Amer. Institute of Math. Sci. AIMS, Springfield, MO (2007).

[4] A. Bressan and F. Rampazzo, On differential systems with vector-valued impulsive controls. Boll. Un. Mat. Ital. B 7 (1988) 641-656.

[5] D.A. Carlson and A. Haurie, Infinite horizon optimal control. Theory and applications. Vol. 290 of Lect. Notes Econom. Math. Systems. Springer-Verlag, Berlin (1987).

[6] P. Cannarsa and G. Da Prato, Nonlinear optimal control with infinite horizon for distributed parameter systems and stationary Hamilton-Jacobi equations. SIAM J. Control and Optim. 27 (1989) 861-875.

[7] P. Cannarsa and C. Sinestrari, Convexity properties of the minimum time function. J. Calc. Var. Partial Differ. Eqs. 3 (1995) 273-298.

[8] F. Da Lio, On the Bellman equation for infinite horizon problems with unbounded cost functional. J. Appl. Math. Optim. 41 (2000) 171-197.

[9] M. Garavello and P. Soravia, Optimality principles and uniqueness for Bellman equations of unbounded control problems with discontinuous running cost. Nonlinear Differ. Equ. Appl. 11 (2004) 271-298.

[10] M. Malisoff, Bounded-from-below solutions of the Hamilton-Jacobi equation for optimal control problems with exit times: vanishing Lagrangians, eikonal equations, and shape-from-shading. Nonlinear Differ. Equ. Appl. 11 (2004) 95-122. 
[11] M. Miller and E.Y. Rubinovich, Impulsive control in continuous and discrete-continuous systems. Kluwer Academic/Plenum Publishers, New York (2003).

[12] M. Motta, Viscosity solutions of HJB equations with unbounded data and characteristic points. Appl. Math. Optim. 4 (2004) $1-26$.

[13] M. Motta and M, F. Rampazzo, State-constrained control problems with neither coercivity nor $L^{1}$ bounds on the controls. Ann. Mat. Pura Appl. 4 (1999) 117-142.

[14] M. Motta and F. Rampazzo, Asymptotic controllability and optimal control. J. Differ. Eqs. 254 (2013) $2744-2763$.

[15] M.Motta and C. Sartori, Exit time problems for nonlinear unbounded control systems. Discrete Contin. Dyn. Syst. 5 (1999) 137-156.

[16] M. Motta and C. Sartori, The value function of an asymptotic exit-time optimal control problem. Nonlinear Differ. Equ. Appl. Springer (2014).

[17] F. Rampazzo and C. Sartori, Hamilton-Jacobi-Bellman equations with fast gradient-dependence. Indiana Univ. Math. J. 49 (2000) 1043-1077.

[18] P. Soravia, Optimality principles and representation formulas for viscosity solutions of Hamilton-Jacobi equations I: Equations of unbounded and degenerate control problems without uniqueness. Adv. Differ. Eqs. (1999) 275-296. 